TRANSACTIONS OF THE

AMERICAN MATHEMATICAL SOCIETY

Volume 361, Number 11, November 2009, Pages 5647-5680

S 0002-9947(09)04672-8

Article electronically published on June 16, 2009

\title{
TRANSVERSE LS CATEGORY FOR RIEMANNIAN FOLIATIONS
}

\author{
STEVEN HURDER AND DIRK TÖBEN
}

\begin{abstract}
We study the transverse Lusternik-Schnirelmann category theory of a Riemannian foliation $\mathcal{F}$ on a closed manifold $M$. The essential transverse category cat $t_{巾}^{e}(M, \mathcal{F})$ is introduced in this paper, and we prove that $\operatorname{cat}_{\pitchfork}^{e}(M, \mathcal{F})$ is always finite for a Riemannian foliation. Necessary and sufficient conditions are derived for when the usual transverse category cat ${ }_{巾}(M, \mathcal{F})$ is finite, and thus cat $e^{e}(M, \mathcal{F})=\operatorname{cat}_{巾}(M, \mathcal{F})$ holds.

A fundamental point of this paper is to use properties of Riemannian submersions and the Molino Structure Theory for Riemannian foliations to transform the calculation of cat $e_{\phi}^{e}(M, \mathcal{F})$ into a standard problem about $\mathbf{O}(q)$ equivariant LS category theory. A main result, Theorem 1.6, states that for an associated $\mathbf{O}(q)$-manifold $\widehat{W}$, we have that cat $e_{\pitchfork}^{e}(M, \mathcal{F})=\operatorname{cat}_{\mathbf{O}(q)}(\widehat{W})$. Hence, the traditional techniques developed for the study of smooth compact Lie group actions can be effectively employed for the study of the LS category of Riemannian foliations.

A generalization of the Lusternik-Schnirelmann theorem is derived: given a $C^{1}$-function $f: M \rightarrow \mathbb{R}$ which is constant along the leaves of a Riemannian foliation $\mathcal{F}$, the essential transverse category cat $t_{\infty}^{e}(M, \mathcal{F})$ is a lower bound for the number of critical leaf closures of $f$.
\end{abstract}

\section{INTRODUCTION}

Let $f: M \rightarrow \mathbb{R}$ be a $C^{1}$-function on a closed Riemannian manifold $M$. We say that $x \in M$ is a critical point if the gradient $\nabla f$ vanishes at $x$. A well-known formula of Lusternik-Schnirelmann [50, 42, 43, 19, gives a lower-bound estimate for the number of critical points,

$$
\#\{x \mid x \in M \text { is critical for } f\} \geq \operatorname{cat}(M),
$$

where $k=\operatorname{cat}(M)$ is the Lusternik-Schnirelmann category of $M$, which is defined as the least number of open sets $\left\{U_{1}, \ldots, U_{k}\right\}$ required to cover $M$ such that each $U_{\ell}$ is contractible in $M$ to a point. The LS category is a measure of the topological complexity of $M$; in the case where $f$ is $C^{2}$ with only Morse-type singularities, the value cat $(M)$ gives a lower bound for the number of cells in a cellular decomposition.

Let $G$ be a compact Lie group and suppose there is a smooth action $M \times G \rightarrow M$ which we can assume preserves a Riemannian metric on $M$. A $G$-invariant set

Received by the editors October 4, 2006

2000 Mathematics Subject Classification. Primary 57R30, 53C12, 55M30; Secondary 57S15.

Key words and phrases. Riemannian foliation, Lusternik-Schnirelmann category, Riemannian submersion, compact Hausdorff foliation, Epstein filtration.

The first author was supported in part by NSF grant DMS-0406254.

The second author was supported by the Schwerpunktprogramm SPP 1154 of the DFG.

(C)2009 American Mathematical Society 
$U \subset M$ is $G$-contractible if there is a $G$-equivariant homotopy $H_{t}: U \rightarrow M$ with $H_{0}$ equal to the inclusion, and such that $H_{1}$ has image in a single $G$-orbit. Define $\operatorname{cat}_{G}(M)$ to be the least number of $G$-invariant open sets $\left\{U_{1}, \ldots, U_{k}\right\}$ required to cover $M$ such that each $U_{\ell}$ is $G$-contractible in $M$ to a single orbit (see Marzantowicz [51] for actions of a compact Lie group, and Ayala, Lasheras and Quintero [3] for proper actions of Lie groups).

A $C^{1}$-function $f: M \rightarrow \mathbb{R}$ is $G$-invariant if $f(x A)=f(x)$ for all $A \in G$; hence the set of critical points $\nabla f=0$ is a closed $G$-invariant set. For a proper $G$ action, each $G$-orbit $x G$ is a closed submanifold of $M$, and Marzantowicz [51] (for $G$ compact) and Ayala, Lasheras and Quintero [3] (for proper actions) showed that the number of critical $G$-orbits is estimated by the $G$-category,

$$
\#\{x G \mid x \in M \text { is critical for } f\} \geq \operatorname{cat}_{G}(M) .
$$

On the other hand, a smooth action of a non-compact Lie group $G$ on a compact manifold is never proper, and the study of its LS-category theory is much more difficult, as the analysis must take into account the dynamical properties of the action. In order that the condition where the critical sets $\nabla f=0$ for the gradient be $G$-invariant, it is useful to assume the action of the group $G$ preserves a Riemannian metric on $M$, and thus the orbits of the action define a singular Riemannian foliation of $M$ [32, 56, 57. If all orbits of the action have the same dimension, then the orbits define a Riemannian foliation of $M$.

In this paper, we study the LS category theory for Riemannian foliations, and prove a Lusternik-Schnirelmann-type estimate for the case of Riemannian foliations.

Theorem 1.1. Let $\mathcal{F}$ be a Riemannian foliation for a compact manifold $M$, and let $f: M \rightarrow \mathbb{R}$ be a $C^{1}$-map which is constant along the leaves of $\mathcal{F}$. A leaf $L_{x}$ of $\mathcal{F}$ through a point $x \in M$ is critical if $\nabla f \mid L_{x}=0$, and hence $\nabla f$ also vanishes on the leaf closure $\bar{L}_{x}$. Then the number of critical leaf closures has a lower bound estimate by the essential transverse $\operatorname{LS}$ category cat $e_{\pitchfork}^{e}(M, \mathcal{F})$ of $\mathcal{F}$,

$$
\#\left\{\bar{L}_{x} \mid x \in M \text { is critical for } f\right\} \geq \operatorname{cat}_{\pitchfork}^{e}(M, \mathcal{F}) .
$$

In the case where all leaves of $\mathcal{F}$ are compact, Colman [12, 18, proved a lower bound estimate for the number of critical leaves in terms of the transverse LS category $\operatorname{cat}_{\pitchfork}(M, \mathcal{F})$, which the estimate (3) generalizes to the general case when $\mathcal{F}$ has non-compact leaves. The transverse $\operatorname{LS}$ category cat ${ }_{\pitchfork}(M, \mathcal{F})$ is infinite when $\mathcal{F}$ has no compact leaves, while the essential transverse $\operatorname{LS}$ category cat $e_{\phi}^{e}(M, \mathcal{F})$ introduced in this paper is always finite.

The basic concept is that of a foliated homotopy: given foliated manifolds $(M, \mathcal{F})$ and $\left(M^{\prime}, \mathcal{F}^{\prime}\right)$, a map $f: M \rightarrow M^{\prime}$ is said to be foliated if for each leaf $L \subset M$ of $\mathcal{F}$, there exists a leaf $L^{\prime} \subset M^{\prime}$ of $\mathcal{F}^{\prime}$ such that $f(L) \subset L^{\prime}$. A $C^{r}$-map $H: M^{\prime} \times[0,1] \rightarrow$ $M$, for $r \geq 0$, is said to be a foliated $C^{r}$-homotopy if $H_{t}$ is foliated for all $0 \leq t \leq 1$, where $H_{t}(x)=H(x, t)$.

Unless otherwise specified, we assume that all maps and homotopies are smooth.

A subset $U \subset M$ is saturated if $U$ is a union of leaves of $\mathcal{F}$. This is the foliation equivalent of the condition for a $G$-action that $U$ be $G$-invariant.

Let $U \subset M$ be an open saturated subset. We say that $U$ is transversely categorical if there is a foliated homotopy $H: U \times[0,1] \rightarrow M$ such that $H_{0}(x)=x$ for all $x \in U$, and $H_{1}: U \rightarrow M$ has image in a single leaf of $\mathcal{F}$. In other words, the 
projection of $U$ to the leaf space $M / \mathcal{F}$ is contractible as a map into a diffeology space [11, 41, 65].

Definition 1.2. The transverse LS category $\operatorname{cat}_{巾}(M, \mathcal{F})$ of a foliated manifold $(M, \mathcal{F})$ is the least number of transversely categorical open saturated sets required to cover $M$. If no such covering exists, then $\operatorname{set}_{\text {cat }}(M, \mathcal{F})=\infty$.

The basic properties of transverse LS category are given in [12, 18. If a foliation $\mathcal{F}$ is defined by a fibration $M \rightarrow B$ over a compact manifold $B$, then $\operatorname{cat}_{\pitchfork}(M, \mathcal{F})=$ $\operatorname{cat}(B)<\infty$, so the LS category of $\mathcal{F}$ agrees with the LS category of the leaf space $M / \mathcal{F}$ in this case. Also, the transverse LS category is an invariant of foliated homotopy. The transverse saturated category $\operatorname{cat}_{\pitchfork}(M, \mathcal{F})$ has been further studied by various authors [14, 15, 17, 38, 40, 47, 70].

The assumption that $\operatorname{cat}_{\pitchfork}(M, \mathcal{F})$ is finite is a strong hypothesis on $\mathcal{F}$, and in particular has consequences for the dynamical properties of $\mathcal{F}$. Recall that a closed subset $X \subset M$ is minimal if $X$ is saturated, and for each leaf $L \subset X$ the closure of $L$ in $M$ equals $K$. By Zorn's Lemma, $M$ compact implies that each closed, saturated subset $X$ contains at least one minimal set.

Now, suppose that $\mathcal{F}$ satisfies $\operatorname{cat}_{\pitchfork}(M, \mathcal{F})<\infty$, and let $\left\{U_{1}, \ldots, U_{k}\right\}$ be a minimal cardinality covering of $M$ by categorical open $\mathcal{F}$-saturated sets, so that $k=\operatorname{cat}_{\pitchfork}(M, \mathcal{F})$. Then each $U_{i}$ contains a compact minimal set $K_{i} \subset U_{i}$ for $\mathcal{F}$. The first author showed in [37] that if $H_{\ell}: U_{\ell} \times[0,1] \rightarrow M$ is a foliated homotopy with $H_{\ell, 0}\left(K_{\ell}\right)$ the inclusion, then the image $H_{\ell, t}\left(K_{\ell}\right)$ is a compact minimal set for all $t$, and in particular $H_{\ell, 1}\left(K_{\ell}\right)$ must be a compact minimal set. Thus, if $H_{\ell}$ is a categorical homotopy, then the image $H_{\ell, 1}$ must be contained in a compact leaf of $L$. If $\mathcal{F}$ has no compact leaves, or simply not enough compact leaves, then a categorical covering of $M$ cannot be found. This result motivates the following definition:

Let $U \subset M$ be an open saturated subset. We say that $U$ is essentially transversely categorical if there is a foliated homotopy $H: U \times[0,1] \rightarrow M$ such that $H_{1}: U \rightarrow M$ has image in a minimal set of $\mathcal{F}$.

Definition 1.3. The essential transverse $L S$ category cat ${ }_{\pitchfork}^{e}(M, \mathcal{F})$ of a foliated manifold $(M, \mathcal{F})$ is the least number of essentially transversely categorical open saturated sets required to cover $M$. If no such covering exists, then set cat $e_{巾}^{e}(M, \mathcal{F})=$ $\infty$.

With this definition, we obtain the following fundamental result:

Theorem 1.4. Let $\mathcal{F}$ be a Riemannian foliation of a compact smooth manifold $M$. Then the essential transverse category $\operatorname{cat}_{巾}^{e}(M, \mathcal{F})$ is finite. If the transverse category $\operatorname{cat}_{巾}(M, \mathcal{F})$ is finite, then $\operatorname{cat}_{巾}(M, \mathcal{F})=\operatorname{cat}_{巾}^{e}(M, \mathcal{F})$.

Moreover, we can give an exact characterization of which Riemannian foliations have finite cat ${ }_{\pitchfork}(M, \mathcal{F})$. Let $L$ be a leaf of $\mathcal{F}$. A foliated isotopy of $L$ is a smooth map $I: L \times[0,1] \rightarrow M$ such that $I_{0}: L \rightarrow M$ is the inclusion of $L$, and for each $0 \leq t \leq 1, I_{t}: L \rightarrow M$ is a diffeomorphism onto its image $L_{t}$, which is a leaf of $\mathcal{F}$. We say that the image leaf $L_{1}$ is foliated isotopic to $L$.

Let $\mathcal{I}_{L}$ denote the set of leaves of $\mathcal{F}$ which are foliated isotopic to $L$. For $x \in M$, we set $\mathcal{I}_{x}=\mathcal{I}_{L_{x}}$.

For an arbitrary foliation, one cannot expect the isotopy classes $\mathcal{I}_{x}$ to have any nice properties at all, and typically one expects that $\mathcal{I}_{x}=L_{x}$. However, for a 
Riemannian foliation, we prove in section 7 that each isotopy class $\mathcal{I}_{x}$ is a smooth submanifold of $M$, and the set of isotopy classes of the leaves of $\mathcal{F}$ defines a Whitney stratification of $M$. A leaf $L_{x}$ (as well as its corresponding stratum $\mathcal{I}_{x}$ ) is said to be locally minimal if $\mathcal{I}_{x}$ is a closed submanifold of $M$.

Theorem 1.5. Let $\mathcal{F}$ be a Riemannian foliation of a compact smooth manifold $M$. Then $\operatorname{cat}_{巾}(M, \mathcal{F})$ is finite if and only if each locally minimal leaf $L_{x}$ is compact, and hence the locally minimal set $\mathcal{I}_{x}$ is a union of compact leaves.

The proofs of Theorems 1.1, 1.4 and 1.5 are based on the very special geometric properties of Riemannian foliations, which were developed by Molino in a series of papers [54, 55, 56, 57]; see also Haefliger [30, 31, 32. We first recall several key facts in order to state our next result; details are given in section 3 .

One of the remarkable corollaries of the Molino structure theory is that for a leaf $L$ of a Riemannian foliation, its closure $\bar{L}$ is a minimal set for $\mathcal{F}$. Thus, the condition in Theorem 1.1 that the critical points of a leafwise constant $C^{1}$-function consists of unions of leaf closures means that in fact they are unions of minimal sets.

Molino's analysis of the geometry and structure of a Riemannian foliation is based on the desingularization of $\mathcal{F}$ using the geometry of a foliated $\mathbf{O}(q)$-bundle: let $\widehat{M} \rightarrow M$ denote the principal $\mathbf{O}(q)$-bundle of orthonormal frames for the normal bundle to $\mathcal{F}$. There exists an $\mathbf{O}(q)$-invariant foliation $\widehat{\mathcal{F}}$ on $\widehat{M}$ whose leaves are the holonomy coverings of the leaves of $\mathcal{F}$, and in particular have the same dimension as the leaves of $\mathcal{F}$. The closures of the leaves of $\widehat{\mathcal{F}}$ are the fibers of an $\mathbf{O}(q)$-equivariant fibration $\widehat{\Upsilon}: \widehat{M} \rightarrow \widehat{W}$, where $\widehat{W}$ is the quotient space by the leaf closures of $\widehat{\mathcal{F}}$, and the $\mathbf{O}(q)$-action on $\widehat{M}$ induces the smooth action of $\widehat{W}$. The quotient space

$$
\widehat{W} / \mathbf{O}(q) \cong W \equiv M / \overline{\mathcal{F}}
$$

is naturally identified with the singular quotient space $W$ of $M$ by the leaf closures of $\mathcal{F}$. Given $w \in \widehat{W}$, the inverse image $\widehat{\pi}^{-1}(w)=\widehat{\widehat{L}}$ is the closure of each leaf $\widehat{L}_{\widehat{x}} \subset \overline{\widehat{L}}$, and the projection of such $\overline{\widehat{L}}$ to $M$ is the closure $\bar{L}$ of a leaf $L_{x}$ of $\mathcal{F}$.

The smooth action of $\mathbf{O}(q)$ on $\widehat{W}$ defines the orbit-type stratification of $\widehat{W}$ in terms of the stabilizer groups of the action, which is one of the key concepts for the study of smooth actions of $\mathbf{O}(q)$ (see [7, 20, 21, 35, 44, 68). A key idea for this work is the use of the associated Whitney stratification, $\widehat{W}=\mathcal{Z}_{1} \cup \cdots \cup \mathcal{Z}_{K}$, where each set $\mathcal{Z}_{\ell}$ is a closed submanifold of $\widehat{W}$ which is $\mathbf{O}(q)$-invariant and such that $\mathcal{Z}_{\ell} / \mathbf{O}(q)$ is connected (see sections 6,0 and 8 ). A stratum $\mathcal{Z}_{\ell}$ is said to be locally minimal if it is a closed submanifold.

Let $\operatorname{cat}_{\mathbf{O}(q)}(\widehat{W})$ denote the $\mathbf{O}(q)$-equivariant category of the space $\widehat{W}$, which is finite as $\widehat{W}$ is compact. The following result, and the techniques developed for its proof, can be considered as the main result of this paper:

Theorem 1.6. Let $\mathcal{F}$ be a Riemannian foliation of a compact manifold $M$. Then

$$
\operatorname{cat}_{\phi}^{e}(M, \mathcal{F})=\operatorname{cat}_{\mathbf{O}(q)}(\widehat{W}) .
$$

Note that for a smooth action of a compact group $G$ on a compact manifold $N$, the $G$-equivariant category of $N$ defined using smooth $G$-homotopies is equal to the $G$-category defined using continuous homotopies, as a continuous homotopy can be 
approximated by a smooth homotopy (Theorem 4.2, Chapter VI of [7]). Thus, the calculation of the equivariant category $\operatorname{cat}_{\mathbf{O}(q)}(\widehat{W})$ is a purely topological problem.

The proof of Theorem 1.6 introduces one of the main new technical ideas of this paper, which can be called the "synchronous lifting property". The choice of a projectable Riemannian metric on $M$, which is $\mathcal{F}$-projectable when restricted to the normal bundle to $\mathcal{F}$, defines a Riemannian metric on the principal $\mathbf{O}(q)$-frame bundle $\pi: \widehat{M} \rightarrow M$ which is then projectable with respect to the foliation $\widehat{\mathcal{F}}$. This projectable Riemannian metric on $T \widehat{M}$ in turn defines a horizontal distribution in $T \widehat{M}$ which is transverse to the fibers of $\pi: \widehat{M} \rightarrow M$. The first key idea is that a homotopy $H: U \times[0,1] \rightarrow M$ on $M$ can be lifted to an $\mathbf{O}(q)$-equivariant $\widehat{\mathcal{F}}$-foliated homotopy of $\widehat{U}=\pi^{-1}(U), \widehat{H}: \widehat{U} \times[0,1] \rightarrow \widehat{M}$. This shows that $\mathcal{F}$-categorical open sets on $M$ are equivalent to $\widehat{\mathcal{F}}$-categorical, $\mathbf{O}(q)$-equivariant categorical sets on $\widehat{M}$.

Similarly, the projection $\widehat{\Upsilon}: \widehat{M} \rightarrow \widehat{W}$ from the frame bundle to the space of leaf closures is a Riemannian submersion, and so has a natural horizontal distribution which is transverse to the projection $\widehat{\Upsilon}$. This is used to show that $\widehat{\mathcal{F}}$-categorical, $\mathbf{O}(q)$-equivariant categorical sets on $\widehat{M}$ are equivalent to $\mathbf{O}(q)$-equivariant categorical sets on $\widehat{W}$. These two procedures combined yields a correspondence between the transversely categorical open sets on $M$ and the $G$-equivariant categorical open sets on $\widehat{W}$. These ideas are developed in detail in sections 3 and 4 .

It is interesting to note that our technique for lifting homotopies to equivariant homotopies, used in both sections 4 and [5] is similar to the method of "averaging isotopies" used in the proof of Theorem 3.1, Chapter VI of 7 .

The remarkable aspect of these arguments is that the connection data is used to define equivariant lifts of the given homotopy; the horizontal distributions are used to "synchronize" the orthonormal frames along the traces of the homotopies. This is possible, even though the homotopy itself need not transform normal frames to normal frames. This technique also has applications to the theory of secondary characteristic classes of $\mathcal{F}$, especially residue theory [39, 48, 49.

This paper can be viewed as a sequel to the work [17] by the first author with Hellen Colman. The results of this paper also extends the results of [15].

The research collaboration of the authors resulting in this work was made possible by the generous support of the second author by the Schwerpunktprogramm SPP 1154 "Globale Differentialgeometrie" of the Deutsche Forschungsgemeinschaft. Both authors are very grateful for this support.

\section{TRAnSVERSE CATEGORY}

We assume that $M$ is a smooth, compact Riemannian manifold without boundary of dimension $m=p+q$, and $\mathcal{F}$ is a smooth Riemannian foliation of dimension $p$ and codimension $q$. Given $x \in M$ we will denote by $L_{x}$ the leaf of $\mathcal{F}$ containing $x$.

Let $\mathcal{E}$ denote the singular Riemannian foliation of $M$ defined by the closures of the leaves of $\mathcal{F}$. (See Molino [56, 57] for properties of $\mathcal{E}$.) The tangential distribution $E=T \mathcal{E}$ is integrable and satisfies the regularity conditions formulated by Stefan [66, 67. Note that all leaves of $\mathcal{E}$ are compact.

The notion of foliated homotopy extends naturally to the case of singular foliations, so that one can define the transverse category cat ${ }_{\pitchfork}(M, \mathcal{E})$ of $\mathcal{E}$. We recall 
two topological lemmas due to Colman [15] which are used to relate the transverse categories of $\mathcal{F}$ and $\mathcal{E}$.

Lemma 2.1. Let $(M, \mathcal{F})$ and $\left(M^{\prime}, \mathcal{F}^{\prime}\right)$ be two foliated manifolds and let $f: M \rightarrow$ $M^{\prime}$ be a foliated continuous map. Let $\mathcal{E}$ denote the partition of $M$ by the closures of the leaves of $\mathcal{F}$, and $\mathcal{E}^{\prime}$ the corresponding partition of $M^{\prime}$. Then $f$ is also $\mathcal{E}$-foliated.

Proof. Let $L \subset M$ be a leaf of $\mathcal{F}$, and $L^{\prime} \subset M^{\prime}$ the leaf of $\mathcal{F}^{\prime}$ such that $f(L) \subset L^{\prime}$. Then $f(\bar{L}) \subset \overline{f(L)} \subset \overline{L^{\prime}}$.

The second lemma is based on the special property of Riemannian foliations that the closure of every leaf in a Riemannian foliation is a minimal set.

Lemma 2.2. Let $\mathcal{F}$ be a Riemannian foliation of a compact manifold $M$. Let $U \subset M$ be an $\mathcal{F}$-saturated open set and $L$ be a leaf of the Riemannian foliation $\mathcal{F}$ such that $L \subset U$. Then $\bar{L} \subset U$.

Proof. Let $L \subset U$, and suppose that the closure $\bar{L}$ is not a subset of $U$. Then there exists a leaf $L^{\prime} \subset \bar{L}$ such that $L^{\prime} \not \subset U$, and as $U$ is saturated, it follows that $L^{\prime} \subset M-U$. The complement $M-U$ is a closed saturated set, so $\overline{L^{\prime}} \subset M-U$. But the fact that $L^{\prime} \subset \bar{L}$ and $\bar{L}$ is a minimal set implies that $\overline{L^{\prime}}=\bar{L}$, so $L \subset \overline{L^{\prime}} \subset M-U$, which is a contradiction.

Proposition 2.3. Let $U \subset M$ be a saturated open set. If $H: U \times[0,1] \rightarrow M$ is an $\mathcal{F}$-homotopy, then $H$ is also an $\mathcal{E}$-homotopy. If $H_{1}$ has image in a single leaf $L \in \mathcal{F}$, or more generally in a minimal set $K$ of $\mathcal{F}$, then $H_{1}$ has image in the leaf $K=\bar{L}$ of $\mathcal{E}$.

Proof. The open set $U$ is an $\mathcal{E}$-saturated set by Lemma 2.2. The map $H_{t}$ is $\mathcal{E}$ foliated by Lemma 2.1. Then the map $H_{1}$ has image in the closure $K=\bar{L}$ by Lemma 2.2

Corollary 2.4. Let $\mathcal{F}$ be a Riemannian foliation of a closed manifold $M$; then

$$
\operatorname{cat}_{\pitchfork}(\mathcal{E}) \leq \operatorname{cat}_{巾}^{e}(\mathcal{F}) \leq \operatorname{cat}_{\pitchfork}(\mathcal{F}) .
$$

\section{Geometry of Riemannian foliations}

The Molino structure theory is a remarkable collection of results about the geometry and topology of a Riemannian foliation on a compact manifold. We recall some of the main results, and in doing so establish the notation which will be used in later sections. The reader should consult Molino [55, 56, 57, Haefliger [30, 31, 32, or Moerdijk and Mrčun [58] for further details, noting that our notation is an amalgam of those used by these authors.

Let $M$ denote a compact, connected smooth manifold without boundary, and $\mathcal{F}$ a smooth Riemannian foliation of codimension $q$, with tangential distribution $T \mathcal{F}$.

Let $g$ denote a Riemannian metric on $T M$ which is projectable with respect to $\mathcal{F}$. Identify the normal bundle $Q$ with the orthogonal space $T \mathcal{F}^{\perp}$, and let $Q$ have the restricted Riemannian metric $g_{Q}=g \mid Q$. For a vector $X \in T_{x} M$ let $X^{\perp} \in Q_{x}$ denote its orthogonal projection.

Given a leafwise path $\gamma$ between points $x, y$ on a leaf $L$, the transverse holonomy $h_{\gamma}$ along $\gamma$ induces a linear transformation $d h_{x}[\gamma]: Q_{x} \rightarrow Q_{y}$. The fact that the 
Riemannian metric $g$ on $T M$ is projectable is equivalent to the fact that the linear holonomy transformation $d h_{x}[\gamma]$ is an isometry for all such paths.

Let $\left\{E_{1}, \ldots, E_{q}\right\}$ be an orthogonal basis for $\mathbb{R}^{q}$. Fix $x \in M$. An orthonormal frame for $Q_{x}$ is an isometric isomorphism $e: \mathbb{R}^{q} \rightarrow Q_{x}$. Let $\operatorname{Fr}\left(Q_{x}\right)$ denote the space of orthogonal frames of $Q_{x}$. Given $e \in \mathbf{F r}\left(Q_{x}\right)$ and $A \in \mathbf{O}(q)$ we obtain a new frame $R_{A}(e)=e A=e \circ A$, where $A: \mathbb{R}^{q} \rightarrow \mathbb{R}^{q}$ is the map induced by matrix multiplication on the left on $\mathbb{R}^{q}$.

The group $\operatorname{Isom}\left(Q_{x}\right)$ of isometries of $Q_{x}$ admits a natural left action on $\operatorname{Fr}\left(Q_{x}\right)$ : for $h_{x} \in \operatorname{Isom}\left(Q_{x}\right)$ and $e \in \operatorname{Fr}\left(Q_{x}\right)$, we define $h_{x} e=h_{x} \circ e: \mathbb{R}^{q} \rightarrow Q_{x}$.

Let $\pi: \widehat{M} \rightarrow M$ denote the bundle of orthonormal frames for $Q$, where the fiber over $x \in M$ is $\pi^{-1}(x)=\operatorname{Fr}\left(Q_{x}\right)$. By the above remarks, $\widehat{M}$ is a principal right-O $(q)$-bundle. We use the notation $\widehat{x}=(x, e) \in \widehat{M}$, where $e \in \mathbf{F r}\left(Q_{x}\right)$.

There is a canonical $\mathbb{R}^{q}$-valued 1-form, the Solder 1-form $\theta: T \widehat{M} \rightarrow \mathbb{R}^{q}$, defined as follows: for $X \in T_{\widehat{x}} \widehat{M}$, then $d_{\widehat{x}} \pi(X) \in T_{x} M$ and set

$$
\theta(X)=e^{-1}\left(d_{\widehat{x}} \pi(X)^{\perp}\right) \in \mathbb{R}^{q} .
$$

Note that for $A \in \mathbf{O}(q), R_{A}^{*} \theta=A^{-1} \circ \theta$.

Let $\nabla$ denote the Levi-Civita connection on $Q \rightarrow M$ defined using the Riemannian metric. Let $\omega: T \widehat{M} \rightarrow \mathfrak{o}(q)$ denote the associated Maurer-Cartan 1-form with values in the matrix Lie algebra $\mathfrak{o}(q)$ of $\mathbf{O}(q)$. Recall that $\omega$ is $A d$-related: for $A \in \mathbf{O}(q), b \in \mathfrak{o}(q)$, and $X \in T \widehat{M}$, we have $A d\left(A^{-1}\right)(b)=A^{-1} b A \in \mathfrak{o}(q)$, and hence

$$
R_{A}^{*}(\omega)=A d\left(A^{-1}\right) \circ \omega ; R_{A}^{*}(\omega)(X)=\omega\left(d R_{A}(X)\right)=A^{-1} \omega(X) A .
$$

Let $\mathcal{H}=\operatorname{ker}(\omega) \subset T \widehat{M}$ denote the horizontal distribution for $\omega$. Then $\mathcal{H}$ is invariant under the right action of $\mathbf{O}(q)$, and for all $\widehat{x} \in \widehat{M}$, the differential $d_{\widehat{x}} \pi: \mathcal{H}_{\widehat{x}} \rightarrow T_{x} M$ is an isomorphism.

Define an $\mathbf{O}(q)$-invariant Riemannian metric $\widehat{g}$ on $T \widehat{M}$ by requiring that the restriction $d \pi: \mathcal{H} \rightarrow T M$ be an isometry and that the fibers of $\pi$ are orthogonal to $\mathcal{H}$. The metric restricted to the fibers is induced by the bi-invariant metric on $\mathbf{O}(q)$ which is defined by the inner product on $\mathfrak{o}(q)$, where $\langle A, B\rangle=\operatorname{Tr}\left(A B^{T}\right)$.

The metric on $T M$ is projectable, therefore the restriction $\nabla^{L}$ to $Q \mid L \rightarrow L$ is a flat connection for each leaf $L \subset M$, so the horizontal distribution of $\nabla^{L}$ is integrable. The inverse image $\pi^{-1}(L) \subset \widehat{M}$ is an $\mathbf{O}(q)$-principal bundle over $L$, and the restricted flat connection $\nabla^{L}$ defines an $\mathbf{O}(q)$-invariant foliation of $\pi^{-1}(L)$, whose leaves cover $L$. The union of all such flat subbundles in $\widehat{M}$ defines an $\mathbf{O}(q)$ invariant foliation $\widehat{\mathcal{F}}$ of $\widehat{M}$ whose tangent distribution $T \widehat{\mathcal{F}}$ is an integrable subbundle of $\mathcal{H}$. The metric on $T \widehat{M}$ is projectable for $\widehat{\mathcal{F}}$, hence $\widehat{\mathcal{F}}$ is also a Riemannian foliation.

The direct sum of the Solder and connection 1-forms, $\theta$ and $\omega$, define a 1-form

$$
\tau \equiv \theta \oplus \omega: T \widehat{M} \longrightarrow \mathbb{R}^{q} \oplus \mathfrak{o}(q) \cong \mathbb{R}^{\left(q^{2}+q\right) / 2}
$$

whose kernel is the distribution $T \widehat{\mathcal{F}}$.

Recall that $\left\{E_{1}, \ldots, E_{q}\right\}$ is an orthonormal basis of $\mathbb{R}^{q}$, and let $\left\{E_{i j} \mid 1 \leq i<\right.$ $j \leq q\}$ denote the corresponding orthonormal basis of $\mathfrak{o}(q)$. Define orthonormal vector fields $\left\{\vec{Z}_{1}, \ldots, \vec{Z}_{q}\right\}$ on $\widehat{M}$ by specifying that at $\widehat{x} \in \widehat{M}$,

$$
\tau\left(\vec{Z}_{i}\right)=\left(E_{i}, 0\right), \vec{Z}_{i}(\widehat{x}) \in T_{\widehat{x}} \widehat{\mathcal{F}}^{\perp} .
$$


Similarly define vector fields $\left\{\vec{Z}_{i j} \mid 1 \leq i<j \leq q\right\}$ by

$$
\tau\left(\vec{Z}_{i j}\right)=\left(0, E_{i j}\right), \vec{Z}_{i j}(\widehat{x}) \in T_{\widehat{x}} \widehat{\mathcal{F}}^{\perp} .
$$

The collection of vector fields $\left\{\vec{Z}_{i}, \vec{Z}_{i j} \mid 1 \leq i<j \leq q\right\}$ span $T_{\widehat{x}} \widehat{\mathcal{F}}^{\perp}$ for each $\widehat{x} \in \widehat{M}$.

Recall that a function $f: \widehat{M} \rightarrow \mathbb{R}$ is $\widehat{\mathcal{F}}$-basic if $f$ is constant on the leaves of $\widehat{\mathcal{F}}$. Let $\mathcal{A}=\mathcal{A}(M, \mathcal{F}, g)$ denote the vector space consisting of all linear combinations

$$
\vec{Z}=\sum_{1 \leq i \leq q} a^{i} \vec{Z}_{i}+\sum_{1 \leq i<j \leq q} b^{i j} \vec{Z}_{i j}
$$

where $\left\{a^{1}, \ldots, a^{q}\right\}$ and $\left\{b^{i j} \mid 1 \leq i<j \leq q\right\}$ are $\widehat{\mathcal{F}}$-basic, smooth functions on $\widehat{M}$.

Let $\mathcal{X}(\widehat{\mathcal{F}})$ denote the smooth vector fields on $\widehat{M}$ that are everywhere tangent to $\widehat{\mathcal{F}}$.

One of the fundamental results of the Molino structure theory is that the flows of the vector fields in $\mathcal{A}$ are foliated:

Proposition 3.1. Let $\vec{Z} \in \mathcal{A}$ and $\vec{X} \in \mathcal{X}(\widehat{\mathcal{F}})$; then $L_{\vec{Z}}(\vec{X}) \in \mathcal{X}(\widehat{\mathcal{F}})$. Hence, for each $t \in \mathbb{R}$, the flow of $\vec{Z}, \Phi_{t}^{\vec{Z}}: \widehat{M} \rightarrow \widehat{M}$, maps leaves of $\widehat{\mathcal{F}}$ to leaves of $\widehat{\mathcal{F}}$.

As the flows of vector fields in $\mathcal{X}(\widehat{\mathcal{F}})$ preserve the leaves of $\widehat{\mathcal{F}}$, it follows that the group of foliated diffeomorphisms $\operatorname{Diff}(\widehat{\mathbf{M}}, \widehat{\mathcal{F}})$ for $\widehat{\mathcal{F}}$ acts transitively on $\widehat{M}$. That is, the foliated manifold $(\widehat{M}, \widehat{\mathcal{F}})$ is transversally complete (TC), and the collection of vector fields $\left\{\vec{Z}_{i}, \vec{Z}_{i j} \mid 1 \leq i<j \leq q\right\}$ define a transverse parallelism (TP) for $\widehat{\mathcal{F}}$.

Given $\widehat{x}=(x, e) \in \widehat{M}$, let $\widehat{L}_{\widehat{x}}$ denote the leaf of $\widehat{\mathcal{F}}$ containing $\widehat{x}$, and let $L_{x}$ be the leaf of $\mathcal{F}$ through $x$. Given a leafwise closed curved $\gamma:[0,1] \rightarrow L$ with $\gamma(0)=\gamma(1)=x$, we have a transverse holonomy map $h_{x}[\gamma]$ which depends only on the homotopy class of $\gamma$. The differential $d h_{x}[\gamma]: Q_{x} \rightarrow Q_{x}$ is an isometry, and so induces a map $d h_{x}: \pi_{1}\left(L_{x}, x\right) \rightarrow \operatorname{Isom}\left(Q_{x}\right)$. Let $\mathcal{K}_{x} \subset \pi_{1}\left(L_{x}, x\right)$ denote the kernel of $d h_{x}$.

The framing $e: \mathbb{R}^{q} \rightarrow Q_{x}$ induces an isomorphism $e^{*}: \operatorname{Isom}\left(Q_{x}\right) \cong \mathbf{O}(q)$, and the composition $d h_{\widehat{x}}=e^{*} \circ d h_{x}: \pi_{1}\left(L_{x}, x\right) \rightarrow \mathbf{O}(q)$ is the framed linear holonomy homomorphism at $\widehat{x}$.

Given $\widehat{x}=(x, e) \in \widehat{M}$, the leaf $\widehat{L}_{\widehat{x}}$ of $\widehat{\mathcal{F}}$ is defined as an integral manifold of the flat connection on the $\mathbf{O}(q)$-frame bundle over $L$, so that $y=(x, f) \in \widehat{L}_{\widehat{x}}$ means that there is $[\gamma] \in \pi_{1}\left(L_{x}, x\right)$ for which $f=d h_{x}[\gamma](e)$. The projection $\pi: \widehat{L}_{\widehat{x}} \rightarrow L_{x}$ is thus the holonomy covering of $L_{x}$ associated to the kernel of $d h_{x}$.

For simplicity of notation, let $\bar{L}_{\widehat{x}}$ denote the closure $\overline{\widehat{L}}_{\widehat{x}}$ of a leaf $\widehat{L}_{\widehat{x}}$ of $\widehat{\mathcal{F}}$. The distinction between $\bar{L}_{\widehat{x}} \subset \widehat{M}$ and the leaf closure $\bar{L}_{x} \subset M$ is indicated by the basepoint.

Recall that the foliation $\widehat{\mathcal{F}}$ is invariant under the right action of $\mathbf{O}(q)$ on $\widehat{M}$. For $\widehat{x} \in \widehat{M}$, define two stabilizer subgroups of $\mathbf{O}(q)$ associated to $\widehat{x}$ :

$$
\begin{aligned}
\mathcal{H}_{\widehat{x}} & \equiv\left\{A \in \mathbf{O}(q) \mid \widehat{L}_{\widehat{x}} A=\widehat{L}_{\widehat{x}}\right\}, \\
\mathbf{H}_{\widehat{x}} & \equiv\left\{A \in \mathbf{O}(q) \mid \bar{L}_{\widehat{x}} A=\bar{L}_{\widehat{x}}\right\} .
\end{aligned}
$$

Clearly, $\mathbf{H}_{\widehat{x}}$ is the topological closure of $\mathcal{H}_{\widehat{x}}$ in $\mathbf{O}(q)$.

Lemma 3.2. There is a natural identification of $\mathcal{H}_{\widehat{x}}$ with the image of $d h_{\widehat{x}}$. 
Proof. Let $\widehat{x}=(x, e)$ and $[\gamma] \in \pi_{1}\left(L_{x}, x\right)$. Set

$$
f \equiv d h_{x}[\gamma](e)=d h_{x}[\gamma] \circ e \in \mathbf{F r}\left(Q_{x}\right), A \equiv e^{-1} \circ f=e^{-1} \circ d h_{x}[\gamma] \circ e \in \mathbf{O}(q) .
$$

Then $\widehat{x} A=(x, e A)=(x, f)$. It follows that for $A=d h_{\widehat{x}}[\gamma]$, we have that $A \in \mathcal{H}_{\widehat{x}}$.

Conversely, if $\widehat{L}_{\widehat{x}} A=\widehat{L}_{\widehat{x}}$, then $\widehat{x} A=(x, e A)=(x, f) \in \widehat{L}_{\widehat{x}}$; hence there exists $[\gamma] \in \pi_{1}\left(L_{x}, x\right)$ such that $f=d h_{x}[\gamma](e)$. Thus, $A=e^{-1} \circ d h_{x}[\gamma] \circ e$ is in the image of $d h_{\widehat{x}}$.

Corollary 3.3. The following are equivalent:

(1) $\mathcal{H}_{\widehat{x}}$ is infinite.

(2) $\mathcal{H}_{\widehat{x}} \subset \mathbf{H}_{\widehat{x}}$ is a proper inclusion.

(3) $\mathcal{K}_{x}$ has infinite index in $\pi_{1}\left(L_{x}, x\right)$.

For $\epsilon>0$, let $\mathbb{D}_{\epsilon}^{q}=\left\{\vec{X} \in \mathbb{R}^{q} \mid\|\vec{X}\|<\epsilon\right\}$. Let $Q^{\epsilon} \rightarrow M$ denote the unit disk subbundle, so that for each $\widehat{x}=(x, e) \in \widehat{M}$, the framing $e$ restricts to an isometry $e: \mathbb{D}_{\epsilon}^{q} \rightarrow Q_{x}^{\epsilon}$.

The lift of $\nabla^{L}$ to $\widehat{Q}=\pi^{*} Q \rightarrow \widehat{L}_{\widehat{x}}$ is also flat, and by the definition of $\widehat{M}$ the leaf $\widehat{L}_{\widehat{x}}$ has trivial holonomy. Thus, combining the isometry $e: \mathbb{D}_{\epsilon}^{q} \rightarrow Q_{x}^{\epsilon}$ with lifted Riemannian connection $\widehat{\nabla}^{L}$ on $\widehat{Q}$ defines an isometric product decomposition

$$
\xi_{\widehat{x}}: \widehat{L}_{\widehat{x}} \times \mathbb{D}_{\epsilon}^{q} \cong \widehat{Q}^{\epsilon} \mid \widehat{L}_{\widehat{x}} .
$$

Let exp: $T M \rightarrow M \times M$ denote the geodesic exponential map, $\pi_{2}: M \times M \rightarrow M$ the projection onto the second factor, and let $\exp _{x}=\pi_{2} \circ \exp : T_{x} M \rightarrow M$ be the exponential map based at $x \in M$. Choose $\epsilon>0$ sufficiently small so that for all $x \in M$, the restriction $\exp _{x}: Q_{x}^{\epsilon} \rightarrow M$ is an embedding.

Proposition 3.4. The composition

$$
\Xi_{\widehat{x}}=\pi_{2} \circ \exp \circ d \pi \circ \xi_{\widehat{x}}: \widehat{L}_{\widehat{x}} \times \mathbb{D}_{\epsilon}^{q} \cong \widehat{Q}^{\epsilon} \longrightarrow M
$$

is a foliated immersion. Given any $\widehat{y} \in \widehat{L}_{\widehat{x}}$ and unit-vector $\vec{X} \in \mathbb{R}^{q}$, the path

$$
\gamma_{(\widehat{x}, \vec{X})}(t)=\Xi_{\widehat{x}}(y, t \vec{X}),-\epsilon<t<\epsilon,
$$

is a unit speed geodesic in $M$ which is orthogonal to $\mathcal{F}$.

Note that Proposition 3.4 does not assert that the map $\Xi_{\widehat{x}}$ is an isometry, as the metric on $\mathbb{D}_{\epsilon}^{q}$ is flat, while the curvature tensor of $M$ transverse to $\mathcal{F}$ need not be zero.

The fundamental group $\pi_{1}\left(L_{x}, x\right)$ acts on the right on $\widehat{L}_{\widehat{x}}$ via covering deck transformations, and acts on $\mathbb{D}_{\epsilon}^{q}$ via the holonomy representation $d h_{x}$. The product action on $\widehat{L}_{\widehat{x}} \times \mathbb{D}_{\epsilon}^{q}$ preserves the product structure, so we obtain a linear foliation $\mathcal{F}^{\omega}$ on the quotient

$$
Q^{\epsilon} \mid L_{x}=\left(\widehat{L}_{\widehat{x}} \times \mathbb{D}_{\epsilon}^{q}\right) / \pi_{1}\left(L_{x}, x\right),(\widehat{y} \cdot \gamma, \vec{X}) \sim\left(\widehat{y}, d h_{x}[\gamma](\vec{X})\right) .
$$

The map $\Xi_{\widehat{x}}$ is constant on the orbits of this action, so we obtain

Corollary 3.5. The induced map

$$
\Xi_{\widehat{x}}: Q^{\epsilon} \mid L_{x} \longrightarrow M
$$

is a foliated immersion. 
Corollary [3.5] implies that a Riemannian foliation has a "linear model" in an open tubular neighborhood of a leaf. This is usually stated for the normal bundle to a compact leaf $L_{x}$ but is equally valid when formulated in terms of immersed submanifolds. The linear foliation (15) and map (16) yields a precise description of the leaves of $\mathcal{F}$ near to $L_{x}$.

For $\widehat{x}=(x, e) \in \widehat{M}$, define the transverse disk to $\mathcal{F}$ as

$$
\iota_{\widehat{x}}: \mathbb{D}_{\epsilon}^{q} \longrightarrow M, \iota_{\widehat{x}}(\vec{X})=\Xi_{\widehat{x}}(\widehat{x}, \vec{X}) .
$$

The image of $\iota_{\widehat{x}}$ will be denoted by $\mathcal{T}_{x}^{\epsilon}$ which is a local transversal to $\mathcal{F}$ through $x$.

Let $y \in \mathcal{T}_{x}^{\epsilon}$ and $\vec{X} \in \mathbb{D}_{\epsilon}^{q}$ so that $\iota_{\widehat{x}}(\vec{X})=y$. Then $L_{y}$ is the image under $\Xi_{\widehat{x}}$ of the leaf $\left(\widehat{L}_{\widehat{x}} \times\{\vec{X}\}\right) / \pi_{1}\left(L_{x}, x\right) \subset Q^{\epsilon} \mid L_{x}$.

Proposition 3.6. The projection $\pi: Q^{\epsilon} \mid L_{x} \rightarrow L_{x}$ is a diffeomorphism when restricted to $L_{y}$ for $y=\iota_{\widehat{x}}(\vec{X})$ if and only if $\vec{X}$ is fixed by all elements of $\mathcal{H}_{\widehat{x}}$.

Proof. $\mathcal{H}_{\widehat{x}}$ is the image of the map $d h_{x}: \pi_{1}\left(L_{x}, x\right) \rightarrow \mathbf{O}(q)$ so that by (16) the covering map $\pi: L_{y} \rightarrow L_{x}$ has fibers isomorphic to the orbit $\vec{X} \cdot \mathcal{H}_{\widehat{x}}$.

Corollary 3.7. Let $y \in \mathcal{T}_{x}^{\epsilon}$. If $\pi: L_{y} \rightarrow L_{x}$ is a diffeomorphism, then there is a 1-parameter family of immersions, $I: L_{x} \times[0,1] \rightarrow M$, such that $I_{t}: L_{x} \rightarrow M$ is a diffeomorphism onto a leaf of $\mathcal{F}$ for all $0 \leq t \leq 1, I_{0}: L_{x} \rightarrow L_{x} \subset M$ is the inclusion of $L_{x}$, and $I_{1}: L_{x} \rightarrow L_{y}$.

Proof. For $z \in L_{x}$, define $I_{t}(z)=\Xi_{\widehat{x}}(\{z\} \times\{t \vec{X}\}), 0 \leq t \leq 1$.

Finally, we recall the aspects of the Molino structure theory for $\mathcal{F}$ which give a complete description of the closures of the leaves of $\mathcal{F}$ and of the foliation induced on them by $\mathcal{F}$.

Theorem 3.8. Let $\mathcal{F}$ be a Riemannian foliation of a closed manifold $M$.

(1) For each $\widehat{x} \in \widehat{M}$, the leaf closure $\bar{L}_{\widehat{x}}$ is a submanifold of $\widehat{M}$, and the set of all such leaf closures defines a foliation $\widehat{\mathcal{E}}$ of $\widehat{M}$ with all leaves compact.

(2) For $\widehat{x}, \widehat{y} \in \widehat{M}$ there exists a foliated diffeomorphism $\Phi_{\widehat{x} \widehat{y}}: \widehat{M} \rightarrow \widehat{M}$ such that $\Phi_{\widehat{x} \widehat{y}}(\widehat{x})=\widehat{y}$; hence $\Phi_{\widehat{x} \widehat{y}}\left(\widehat{L}_{\widehat{x}}\right)=\widehat{L}_{\widehat{y}}$ and $\Phi_{\widehat{x} \widehat{y}}\left(\bar{L}_{\widehat{x}}\right)=\bar{L}_{\widehat{y}}$

(3) There exists a closed manifold $\widehat{W}$ with a right $\mathbf{O}(q)$-action, and an $\mathbf{O}(q)$ equivariant fibration $\widehat{\Upsilon}: \widehat{M} \rightarrow \widehat{W}$ whose fibers of $\widehat{\Upsilon}$ are the leaves of $\widehat{\mathcal{E}}$.

(4) The metric on $T \widehat{M}$ defined above is projectable for the foliation $\widehat{\mathcal{E}}$, and for the induced metric on $T \widehat{W}$ the fibration $\widehat{\Upsilon}: \widehat{M} \rightarrow \widehat{W}$ is a Riemannian submersion.

Let $W=M / \overline{\mathcal{F}}$ denote the Hausdorff space defined as the quotient of $M$ by the closures of the leaves of $\mathcal{F}$, and $\Upsilon: M \rightarrow W$ the quotient map. Then there is an $\mathbf{O}(q)$-equivariant commutative diagram:

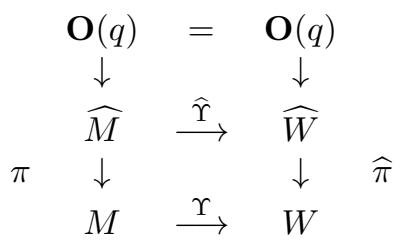

Note that given $\widehat{x} \in \widehat{M}$ and $\widehat{w}=\widehat{\Upsilon}(\widehat{x})$, we have $\bar{L}_{\widehat{x}}=\widehat{\Upsilon}^{-1}(\widehat{w})$. 
Corollary 3.9. Let $\widehat{x}=(x, e)$ and let $L_{x}$ be the leaf of $\mathcal{F}$ through $x$. Then

$$
\bar{L}_{x}=\pi\left(\bar{L}_{\widehat{x}}\right)=\bar{L}_{\widehat{x}} / \mathbf{H}_{\widehat{x}} .
$$

The restriction $\pi: \bar{L}_{\widehat{x}} \rightarrow \bar{L}_{x}$ is a principal $\mathbf{H}_{\widehat{x}}$-fibration and is a covering map if and only if $\mathbf{H}_{\widehat{x}}$ is a finite group.

Let $\mathcal{W}$ be the horizontal distribution for the Riemannian submersion $\widehat{\Upsilon}: \widehat{M} \rightarrow$ $\widehat{W}$. Then $\mathcal{W} \subset T \widehat{M}$ is the subbundle of vectors orthogonal to the fibers of $\widehat{\Upsilon}: \widehat{M} \rightarrow$ $\widehat{W}$. Note that $T \widehat{\mathcal{F}}$ is contained in the kernel of $d \widehat{\Upsilon}$, so that $\mathcal{W} \subset T \widehat{\mathcal{F}}^{\perp}$ and each leaf $\widehat{L}_{\widehat{x}}$ is orthogonal to the fibers of $\pi: \widehat{M} \rightarrow M$.

The second part of the Molino structure theory concerns the geometry of $\bar{L}_{\widehat{x}}$ with the foliation defined by the leaves of $\widehat{\mathcal{F}}$.

Theorem 3.10. There exists a connected, simply connected Lie group G with Lie algebra $\mathfrak{g}$ such that the restricted foliation $\widehat{\mathcal{F}}$ on $\bar{L}_{\widehat{x}}$ is a Lie $G$-foliation with all leaves dense, defined by a $\mathfrak{g}$-valued connection 1 -form $\omega_{\mathfrak{g}}: T \bar{L}_{\widehat{y}} \longrightarrow \mathfrak{g}$.

Moreover, given $\widehat{x} \in \widehat{M}$ and a contractible open neighborhood $\widehat{V} \subset \widehat{W}$ of $\widehat{w}=\widehat{\pi}(\widehat{x})$ there exists an $\widehat{\mathcal{F}}$-foliated diffeomorphism

$$
\Phi_{\widehat{x}}: \bar{L}_{\widehat{x}} \times \widehat{V} \longrightarrow \widehat{U}=\widehat{\pi}^{-1}(\widehat{V}) \subset \widehat{M} .
$$

Hence, $\widehat{\mathcal{F}} \mid \widehat{U}$ is defined by a $\mathfrak{g}$-valued connection $1-$ form $\omega_{\mathfrak{g}}^{\widehat{U}}: T \widehat{U} \longrightarrow \mathfrak{g}$.

\section{Equivariant Foliated transverse CATEgory}

In this section, we introduce the $\mathbf{O}(q)$-transverse category of $\widehat{\mathcal{F}}$ and show that this is equal to the transverse category of $\mathcal{F}$. The proof uses the horizontal distribution of a projectable metric on $M$ and Molino theory, with techniques analogous to those used in the study of foliations with an Ehresmann connection [5, 6].

Let $\widehat{U} \subset \widehat{M}$ be an $\mathbf{O}(q)$-invariant, $\widehat{\mathcal{F}}$-saturated open subset. Let $\widehat{H}: \widehat{U} \times[0,1] \rightarrow$ $\widehat{M}$ be an $\mathbf{O}(q)$-equivariant, $\widehat{\mathcal{F}}$-foliated homotopy. Then for $U=\pi(\widehat{U}), \widehat{H}$ descends to an $\mathcal{F}$-foliated homotopy $H: U \times[0,1] \rightarrow M$. The following result proves the converse:

Proposition 4.1. Let $H: U \times[0,1] \rightarrow M$ be an $\mathcal{F}$-foliated homotopy with $H_{0}(x)=$ $x$, for $x \in U$. Then there exists an $\mathbf{O}(q)$-equivariant, $\widehat{\mathcal{F}}$-foliated homotopy

$$
\widehat{H}: \widehat{U} \times[0,1] \rightarrow \widehat{M}
$$

such that $\pi \circ \widehat{H}=H \circ(\pi \times I d)$. That is, the following diagram commutes:

$$
\pi \times I d \quad \begin{array}{ccccc} 
& \widehat{U} \times[0,1] & \stackrel{\widehat{H}}{\longrightarrow} & \widehat{M} & \\
& \downarrow & & \downarrow & \pi \\
& U \times[0,1] & \stackrel{H}{\longrightarrow} & M &
\end{array}
$$

Proof. Recall that $\mathcal{H}=\operatorname{ker}(\omega) \subset T \widehat{M}$ is the horizontal distribution for the basic connection $\omega$. For each $\widehat{y}=(e, f) \in \widehat{M}, d \pi: \mathcal{H}_{\widehat{y}} \rightarrow T_{y} M$ is an isomorphism.

Given $\widehat{x}=(x, e) \in \widehat{U}$, let $\gamma_{x}(t)=H(x, t)$ denote the path traced out in $M$ by the homotopy. Let $\widehat{\gamma}_{\widehat{x}}:[0,1] \rightarrow \widehat{M}$ denote the horizontal lift of $\gamma_{x}(t)$ starting at $\widehat{x}$. That is, $\widehat{\gamma}_{\widehat{x}}^{\prime}(t) \in \mathcal{H}$ and $d \pi\left(\widehat{\gamma}_{\widehat{x}}^{\prime}(t)\right)=\gamma_{x}^{\prime}(t)$ for all $0 \leq t \leq 1$. Define $\widehat{H}(\widehat{x}, t)=\widehat{\gamma}_{\widehat{x}}(t)$.

The map $\widehat{H}$ is smooth, as the lift of the path $\gamma_{x}$ to the solution curve $\widehat{\gamma}_{\widehat{x}}(t)$ depends smoothly on the initial conditions $\widehat{x}$. 
Given $A \in \mathbf{O}(q)$ set $f=e A$ and $\widehat{y}=(x, f)$. Then $R_{A}\left(\widehat{\gamma}_{\widehat{x}}(0)\right)=\widehat{y}$,

$$
\begin{aligned}
R_{A}\left(\widehat{\gamma}_{\widehat{x}}(t)\right)^{\prime} & =d R_{a}\left(\widehat{\gamma}_{\widehat{x}}^{\prime}(t)\right) \in d R_{A} \mathcal{H}=\mathcal{H}, \\
d \pi\left(d R_{A}\left(\widehat{\gamma}_{\widehat{x}}^{\prime}(t)\right)\right) & =\left(\pi\left(R_{A}\left(\widehat{\gamma}_{\widehat{x}}\right)\right)\right)^{\prime}(t)=\left(\pi\left(\widehat{\gamma}_{\widehat{x}}\right)\right)^{\prime}(t)=\gamma_{x}^{\prime}(t),
\end{aligned}
$$

so by uniqueness we have $R_{A}\left(\widehat{\gamma}_{\widehat{x}}(t)\right)=\widehat{\gamma}_{\widehat{y}}(t)$. Thus, $\widehat{H}$ is $\mathbf{O}(q)$-equivariant.

It remains to show that $\widehat{H}$ is $\widehat{\mathcal{F}}$-foliated.

Let $\mathcal{I}^{p}=(-1,1)^{p}, \mathcal{I}^{q}=(-1,1)^{q}$ and $\mathcal{I}^{m}=(-1,1)^{m}$, where $m=p+q$.

Given $x \in U$, let $\varphi: V \rightarrow \mathcal{I}^{m}$ be a foliation chart such that $x \in V \subset U$. The connected components of the leaves of $\mathcal{F} \mid V$ are the plaques

$$
\mathcal{P}_{\xi}=\varphi^{-1}\left(\mathcal{I}^{p} \times\{\xi\}\right), \xi \in \mathcal{I}^{q} .
$$

Let $\varphi_{t r}: U \rightarrow \mathcal{I}^{q}$ denote the projection onto the transverse coordinate $\xi$. By assumption, the restriction of $g$ to $Q \mid U$ projects to a Riemannian metric $g_{U}$ on $\mathcal{I}^{q}$.

Let $\widehat{\mathcal{I}^{q}} \rightarrow \mathcal{I}^{q}$ denote the $\mathbf{O}(q)$-bundle of orthogonal frames of $T \mathcal{I}^{q}$, and $\widehat{\pi}: \widehat{\mathcal{I}^{q}} \rightarrow$ $\mathcal{I}^{q}$ the projection. Let $\omega_{U}: \widehat{\mathcal{I}^{q}} \rightarrow \mathfrak{o}(q)$ be the Levi-Civita connection 1-form for $g_{U}$, with horizontal distribution $\mathcal{H}_{U} \subset T \widehat{\mathcal{I} q}$.

The assumption that $g$ is projectable implies that the restriction to $\widehat{U}=\pi^{-1}(U)$ of the connection 1-form $\omega$ for $Q$ satisfies $\omega \mid \widehat{U}=\varphi_{t r}^{*}\left(\omega_{U}\right)$. In particular, this implies that the horizontal distribution $\mathcal{H}$ contains the tangent vectors to the fibers of the projection map $\widehat{\varphi_{t r}}: \widehat{U} \rightarrow \widehat{\mathcal{I}^{q}}$. Actually, this fact is obvious, as the fibers are exactly the plaques of $\widehat{\mathcal{F}} \mid U$. The following technical result is used to show that $\widehat{H}$ is a foliated map.

Lemma 4.2. Assume we are given smooth maps $\widehat{G}:[0, \delta] \times[0, \epsilon] \rightarrow \widehat{U}$ and $G:[0, \delta]$ $\times[0, \epsilon] \rightarrow U$ such that $\pi \circ \widehat{G}=G$. For each $0 \leq s \leq \delta$, define the smooth curves $\sigma_{s}(t)=G(s, t)$ and $\widehat{\sigma}_{s}(t)=\widehat{G}(s, t)$. Further assume that

(1) for each $0 \leq t \leq \epsilon$, the curve $\gamma_{t}$ defined by $\gamma_{t}(s)=G(s, t)$ is contained in a leaf of $\mathcal{F} \mid U$,

(2) the curve $\widehat{\gamma}_{0}$ defined by $\widehat{\gamma}_{0}(s)=\widehat{G}(s, 0)$ is contained in a leaf of $\widehat{\mathcal{F}} \mid \widehat{U}$,

(3) for all $(s, t) \in[0, \delta] \times[0, \epsilon]$, the tangent vector $\widehat{\sigma}_{s}^{\prime}(t) \in \mathcal{H} \mid \widehat{U}$.

Then the curve $\widehat{\gamma}_{t}$ defined by $\widehat{\gamma}_{t}(s)=\widehat{G}(s, t)$ is contained in a leaf of $\widehat{\mathcal{F}} \mid \widehat{U}$.

Proof. Consider the diagram

$$
\begin{array}{rlcccccc}
{[0, \delta] \times[0, \epsilon]} & \stackrel{\widehat{G}}{\longrightarrow} & \widehat{U} & & & \widehat{\varphi_{t r}} & \widehat{\mathcal{I}^{q}} & \\
& & \downarrow & \pi & & & \downarrow & \widehat{\pi} \\
(s, t) \in[0, \delta] \times[0, \epsilon] & \stackrel{G}{\longrightarrow} & U & & \stackrel{\varphi_{t r}}{\longrightarrow} & \mathcal{I}^{q} &
\end{array}
$$

By Lemma 4.2 $(1)$ the composition $\tau_{s}(t)=\varphi_{t r} \circ G(s, t)$ is constant in $s$, and thus defines a smooth path $\tau:[0, \epsilon] \rightarrow \mathcal{I}^{q}, \tau(t)=\tau_{s}(t)$ for any choice of $0 \leq s \leq \delta$. Let $\widehat{\tau}:[0, \epsilon] \rightarrow \widehat{\mathcal{I}^{q}}$ be the lift of $\tau$ to a horizontal path with respect to $\omega_{U}$.

For $0 \leq s \leq \delta$, then $\widehat{\sigma}_{s}^{\prime}(t) \in \mathcal{H} \mid \widehat{U}$ by Lemma $4.2(3)$. Hence $d \widehat{\varphi_{t r}}\left(\widehat{\sigma}_{s}^{\prime}(t)\right)=$ $\left(\widehat{\varphi_{t r}} \circ \widehat{\sigma}_{s}\right)^{\prime}(t)$ is horizontal for $\omega_{U}$.

The assumption $\pi \circ \widehat{G}=G$ implies $d \pi\left(\widehat{\sigma}_{s}^{\prime}(t)\right)=\sigma_{s}^{\prime}(t)$, hence

$$
d \widehat{\pi}\left(\widehat{\varphi_{t r}} \circ \widehat{\sigma}_{s}\right)^{\prime}(t)=\tau_{s}^{\prime}(t)=\tau^{\prime}(t) .
$$

Thus, the curve $\widehat{\varphi_{t r}} \circ \widehat{\sigma}_{s}(t)$ is a horizontal lift of $\tau(t)$, with initial point $\widehat{\varphi_{t r}} \circ \widehat{\sigma}_{s}(0)$.

The initial point $\widehat{\varphi_{t r}} \circ \widehat{\sigma}_{s}(0)$ is independent of $s$ by Lemma 4.2(2); thus it follows that the curve $\widehat{\varphi_{t r}} \circ \widehat{\sigma}_{s}(t)$ is independent of $s$. 
That is, for all $0 \leq t \leq \epsilon$, the curve $s \mapsto \widehat{\sigma}_{s}(t)$ is contained in a fixed fiber of $\widehat{\varphi t r}$, which is a plaque of $\widehat{\mathcal{F}} \mid \widehat{\widehat{U}}$, and so is contained in a leaf of $\widehat{\mathcal{F}}$.

We now complete the proof of Proposition 4.1. Let $\widehat{x} \in \widehat{U}$ and $\widehat{y} \in \widehat{L}_{\widehat{x}} \cap \widehat{U}$ be in the plaque containing $\widehat{x}$. We must show that $\widehat{H}_{t}(\widehat{x})$ and $\widehat{H}_{t}(\widehat{y})$ lie in the same leaf for all $0 \leq t \leq 1$.

Choose a smooth path $\widehat{\gamma}_{\widehat{x} \widehat{y}}:[0,1] \rightarrow \widehat{L}_{\widehat{x}} \cap \widehat{U}$ with $\widehat{\gamma}_{\widehat{x} \widehat{y}}(0)=\widehat{x}$ and $\widehat{\gamma}_{\widehat{x} \widehat{y}}(1)=\widehat{y}$. Define $x=\pi(\widehat{x}), y=\pi(\widehat{y})$, and set $\gamma_{x y}(s)=\pi\left(\widehat{\gamma}_{\widehat{x} \widehat{y}}(s)\right)$ so that $\gamma_{x y}$ is a smooth path in $L_{x} \cap U$ from $x$ to $y$.

Compose these paths with the given homotopy $H$ and its lift $\widehat{H}$ to obtain maps

$$
\begin{aligned}
\widehat{H}(s, t) & =\widehat{H}\left(\widehat{\gamma}_{\widehat{x} \widehat{y}}(s), t\right), \\
H(s, t) & =H\left(\gamma_{x y}(s), t\right) .
\end{aligned}
$$

The maps $H_{0}$ and $\widehat{H}_{0}$ are inclusions, so $\widehat{H}(s, 0)=\widehat{\gamma}_{\widehat{x} \widehat{y}}(s)$ and $H(s, 0)=\gamma_{x y}(s)$ for $0 \leq s \leq 1$. We are also given that $\pi \circ \widehat{H}=H$, so that $\pi \circ \widehat{H}(s, t)=H(s, t)$ for all $0 \leq s \leq 1,0 \leq t \leq 1$.

If the image of $H:[0,1] \times[0,1] \rightarrow M$ is contained in a foliation chart $\varphi: V \rightarrow$ $\mathcal{I}^{p} \times \mathcal{I}^{q}$, then we can directly apply Lemma 4.2 to obtain the claim.

For the general case, observe that there exists an integer $N>0$ so that for $s_{\mu}=\mu / N$ and $t_{\nu}=\nu / N$ for each $0 \leq \mu, \nu<N$ there is a foliation chart $\varphi_{\mu \nu}: V_{\mu \nu} \rightarrow$ $\mathcal{I}^{p} \times \mathcal{I}^{q}$ such that $H\left(\left[s_{\mu}, s_{\mu+1}\right] \times\left[t_{\nu}, t_{\nu+1}\right]\right) \subset V_{\mu \nu}$.

Set $\delta=\epsilon=1 / N$, and define for $0 \leq s, t \leq 1 / N$

$$
\widehat{G}_{\mu \nu}(s, t)=\widehat{H}\left(s_{\mu}+s, t_{\nu}+t\right), G_{\mu \nu}(s, t)=H\left(s_{\mu}+s, t_{\nu}+t\right) .
$$

For $\nu=0$ and each $0 \leq \mu<N$, the maps $\widehat{G}_{\mu 0}, G_{\mu 0}$ satisfy the hypotheses of Lemma 4.2. The conclusion of Lemma 4.2 implies that this is again true for $\nu=1$, so that one can apply Lemma 4.2 repeatedly to obtain that the curve $s \rightarrow \widehat{H}(s, 1)$ lies in a leaf of $\widehat{\mathcal{F}}$ as claimed.

Definition 4.3. Let $\widehat{U} \subset \widehat{M}$ be an $\mathbf{O}(q)$-invariant, $\widehat{\mathcal{F}}$-saturated open subset. We say that $\widehat{U}$ is $\mathbf{O}(q)$-transversely categorical if there exists an $\mathbf{O}(q)$-equivariant, $\widehat{\mathcal{F}}$ foliated homotopy $\widehat{H}: \widehat{U} \times[0,1] \rightarrow \widehat{M}$ such that $\widehat{H}_{0}$ is the inclusion and $\widehat{H}_{1}$ has image in the orbit $\bar{L}_{\widehat{x}} \cdot \mathbf{O}(q)$ of the closure $\bar{L}_{\widehat{x}}$ of a leaf $L_{\widehat{x}}$ of $\widehat{\mathcal{F}}$.

The $\mathbf{O}(q)$-transverse category of $\widehat{\mathcal{F}}$, denoted by $\operatorname{cat}_{\mathbf{O}(q)}(\widehat{M}, \widehat{\mathcal{F}})$, is the least number of $\mathbf{O}(q)$ transversely categorical sets required to cover $\widehat{M}$. We then have:

Corollary 4.4. Let $\mathcal{F}$ be a Riemannian foliation of a compact manifold $M$; then

$$
\operatorname{cat}_{\phi}^{e}(M, \mathcal{F})=\operatorname{cat}_{\mathbf{O}(q)}(\widehat{M}, \widehat{\mathcal{F}}) .
$$

\section{5. $\mathbf{O}(q)$-EQUIVARIANT CATEGORY}

In this section, we show that the $\mathbf{O}(q)$-transverse category $\operatorname{cat}_{\mathbf{O}(q)}(\widehat{M}, \widehat{\mathcal{F}})$ of $\widehat{\mathcal{F}}$ is equal to the $\mathbf{O}(q)$-equivariant category $\operatorname{cat}_{\mathbf{O}(q)}(\widehat{W})$ of $\widehat{W}$, which will complete the proof of Theorem[1.6. The proof uses the horizontal distribution of the Riemannian submersion $\widehat{\Upsilon}: \widehat{M} \rightarrow \widehat{W}$ defined by the projectable metric for $\widehat{\mathcal{F}}$.

Let $\widehat{U} \subset \widehat{M}$ be an $\mathbf{O}(q)$-invariant, $\widehat{\mathcal{F}}$-saturated open subset. Suppose that $\widehat{H}: \widehat{U} \times[0,1] \rightarrow \widehat{M}$ is an $\mathbf{O}(q)$-equivariant, $\widehat{\mathcal{F}}$-foliated homotopy. Then by Proposition 2.3 applied to $\widehat{\mathcal{F}}$, we obtain an $\mathbf{O}(q)$-invariant open set $\mathcal{U}=\widehat{\Upsilon}(\widehat{U}) \subset \widehat{W}$ and 
an $\mathbf{O}(q)$-equivariant homotopy $\mathcal{H}: \mathcal{U} \times[0,1] \rightarrow \widehat{W}$ which is the quotient map of $\widehat{H}$. Conversely, we have:

Proposition 5.1. Let $\mathcal{U} \subset \widehat{W}$ be an $\mathbf{O}(q)$-invariant open subset. Given an $\mathbf{O}(q)$ equivariant homotopy $\mathcal{H}: \mathcal{U} \times[0,1] \rightarrow \widehat{W}$ with $\mathcal{H}(\widehat{x}, 0)=\widehat{x}$, for $\widehat{x} \in \mathcal{U}$, set $\widehat{U}=$ $\widehat{\Upsilon}^{-1}(\mathcal{U}) \subset \widehat{M}$. Then there is an $\mathbf{O}(q)$-equivariant, $\widehat{\mathcal{F}}$-foliated homotopy $\widehat{H}: \widehat{U} \times$ $[0,1] \rightarrow \widehat{M}$ such that $\widehat{\Upsilon} \circ \widehat{H}=\mathcal{H} \circ(\widehat{\Upsilon} \times I d)$.

Proof. For each $(\widehat{w}, t) \in \mathcal{U} \times[0,1]$, set $\widehat{w}_{t}=\mathcal{H}(\widehat{w}, t)$, and let

$$
\mathcal{H}^{\prime}(\widehat{w}, t)=\frac{d}{d t} \mathcal{H}(\widehat{w}, t) \in T_{\widehat{w}_{t}} \widehat{W}
$$

denote the tangent vector along the time coordinate.

For $A \in \mathbf{O}(q)$, let $R_{A}: \widehat{W} \rightarrow \widehat{W}$ denote the right action $R_{A}(\widehat{w})=\widehat{w} A$. The assumption that $\mathcal{H}$ is $\mathbf{O}(q)$-invariant implies

$$
\mathcal{H}^{\prime}(\widehat{w} A, t)=\frac{d}{d t}\left(R_{A} \mathcal{H}(\widehat{w}, t)\right)=d R_{A}\left(\mathcal{H}^{\prime}(\widehat{w}, t)\right)
$$

so that $\mathcal{H}^{\prime}(\widehat{w}, t)$ is an $\mathbf{O}(q)$-invariant vector field.

Recall that $\mathcal{W} \subset T \widehat{M}$ is the subbundle of vectors orthogonal to the fibers of $\widehat{\Upsilon}: \widehat{M} \rightarrow \widehat{W}$. Then $\mathcal{W}$ is $\mathbf{O}(q)$-invariant, as the Riemannian metric $\widehat{g}$ on $T \widehat{M}$ is $\mathbf{O}(q)$-invariant, and $\widehat{\Upsilon}$ is $\mathbf{O}(q)$-equivariant.

For $\widehat{x} \in \widehat{U}$ with image $\widehat{w}=\widehat{\pi}(\widehat{x})$, define a smooth curve $\widehat{x}_{t}:[0,1] \rightarrow \widehat{M}$ by requiring that

$$
\widehat{x}_{0}=\widehat{x}, \widehat{x}_{t}^{\prime}=\frac{d \widehat{x}_{t}}{d t} \in \mathcal{W}, d \widehat{\Upsilon}\left(\widehat{x}_{t}^{\prime}\right)=\mathcal{H}^{\prime}(\widehat{w}, t)=\widehat{w}_{t}^{\prime} .
$$

Thus, $\widehat{x}_{t}$ is an integral curve for the horizontal distribution $\mathcal{W}$. Define $\widehat{H}(\widehat{x}, t)=\widehat{x}_{t}$.

It follows from (28) that $\widehat{\Upsilon}\left(\widehat{x}_{t}\right)=\widehat{w}_{t}$, hence $\widehat{\Upsilon} \circ \widehat{H}=\mathcal{H} \circ(\widehat{\Upsilon} \times I d)$.

The function $\widehat{H}$ is smooth, as the integral curves $\widehat{x}_{t}$ depend smoothly on the initial condition $\widehat{x}_{0}=\widehat{x}$. The function $\widehat{H}$ is $\mathbf{O}(q)$-equivariant, as given $A \in \mathbf{O}(q)$

and

$$
\frac{d}{d t} R_{A}\left(\widehat{x}_{t}\right)=d R_{A}\left(\widehat{x}_{t}^{\prime}\right) \in d R_{A}(\mathcal{W})=\mathcal{W}
$$

$$
d \widehat{\Upsilon}\left(\frac{d}{d t} R_{A}\left(\widehat{x}_{t}\right)\right)=d \widehat{\Upsilon}\left(d R_{A}\left(\widehat{x}_{t}^{\prime}\right)\right)=d R_{A}\left(d \widehat{\Upsilon}\left(\widehat{x}_{t}^{\prime}\right)\right)=\mathcal{H}^{\prime}(\widehat{x} A, t) .
$$

It remains to show that $\widehat{H}_{t}: \widehat{U} \rightarrow \widehat{M}$ is $\widehat{\mathcal{F}}$-foliated. We will show that for each vector field $\vec{X} \in \mathcal{X}(\widehat{\mathcal{F}})$ tangent to $\widehat{\mathcal{F}}$ and for $0 \leq t \leq 1, d \widehat{H}_{t}(\vec{X}) \in \mathcal{X}(\widehat{\mathcal{F}})$.

Let $\Phi_{s}^{\vec{X}}: \widehat{M} \rightarrow \widehat{M}$ denote the flow of $\vec{X}$, which preserves the leaves of $\widehat{\mathcal{F}}$. The flow $\Phi_{s}^{\vec{X}}$ also preserves the leaf closures of $\widehat{\mathcal{F}}$, so induces a quotient flow on $\widehat{W}$ which is constant. The metric $\widehat{g}$ on $T \widehat{M}$ is $\widehat{\mathcal{F}}$-projectable, hence its projection to $T \widehat{\mathcal{F}}^{\perp}$ is invariant under the flow $\Phi_{s}^{\vec{X}}$.

For each $\widehat{x} \in \widehat{M}$, the trace $t \mapsto \widehat{x}_{t}=\widehat{H}_{t}(x)$ is determined by the flow of the non-autonomous vector field $\widehat{H}^{\prime}=\widehat{H}^{\prime}(\widehat{x}, t)$ which is $\widehat{\Upsilon}$-related to the vector field $\mathcal{H}^{\prime}=\mathcal{H}^{\prime}(\widehat{w}, t)$. Set $\widehat{x}_{t, s}=\Phi_{s}^{\vec{X}}\left(\widehat{x}_{t}\right)$. Note that $\widehat{\Upsilon}\left(\widehat{x}_{t, s}\right)$ is constant as a function of $s$.

Apply $d \Phi_{s}^{\vec{X}}$ to the $\widehat{\Upsilon}$-related vector field $\widehat{x}_{t}^{\prime}=\widehat{H}^{\prime}(\widehat{x}, t)$ to obtain

$$
\vec{Y}_{t, s}=d \Phi_{s}^{\vec{X}}\left(\widehat{H}^{\prime}(\widehat{x}, t)\right)=d \Phi_{s}^{\vec{X}}\left(\widehat{x}_{t}^{\prime}\right) .
$$


Note that $\left.\frac{d}{d s}\right|_{s=0} \vec{Y}_{t, s}=L_{\vec{X}} H^{\prime}(\widehat{x}, t)$. We claim that the Lie bracket

$$
\left(L_{\vec{X}} H^{\prime}\right)_{\widehat{x}_{t}}=\left[\vec{X}, \widehat{H}^{\prime}\right]_{\widehat{x}_{t}} \in T_{\widehat{x}_{t}} T \widehat{\mathcal{F}},
$$

which implies that the flow of $\widehat{H}^{\prime}$ preserves the foliation $\widehat{\mathcal{F}}$.

At each point $\widehat{x}_{t, s} \in \widehat{M}$ there is an orthogonal decomposition

$$
T_{\widehat{x}_{t, s}} \widehat{M}=T_{\widehat{x}_{t, s}}^{\mathcal{F}} \widehat{M} \oplus T_{\widehat{x}_{t, s}}^{\widehat{\Upsilon}} \widehat{M} \oplus \mathcal{W}_{\widehat{x}_{t, s}},
$$

where $T_{\widehat{x}_{t, s}}^{\mathcal{F}} \widehat{M}=T_{\widehat{x}_{t, s}} \widehat{\mathcal{F}}$, and $T_{\widehat{x}_{t, s}}^{\widehat{\Upsilon}} \widehat{M}=T_{\widehat{x}_{t, s}} \bar{L}_{\widehat{x}_{t, s}} \cap T_{\widehat{x}_{t, s}} \widehat{\mathcal{F}}^{\perp}$ consists of the vectors orthogonal to $T_{\widehat{x}_{t, s}} \widehat{\mathcal{F}}$ and tangent to the fibers of $\widehat{\Upsilon}$. We decompose $\vec{Y}_{t, s}$ into its components,

$$
\vec{Y}_{t, s}=\vec{Y}_{t, s}^{\widehat{\mathcal{F}}}+\vec{Y}_{t, s}^{\widehat{\Upsilon}}+\vec{Y}_{t, s}^{\mathcal{W}}
$$

The projection $d \widehat{\Upsilon}\left(\vec{Y}_{t, s}\right)$ is constant as a function of $s$, so

$$
d \widehat{\Upsilon}\left(\frac{d}{d s} \vec{Y}_{t, s}^{\mathcal{W}}\right)=\frac{d}{d s} d \widehat{\Upsilon}\left(\vec{Y}_{t, s}^{\mathcal{W}}\right)=\frac{d}{d s} d \widehat{\Upsilon}\left(\vec{Y}_{t, s}\right)=0,
$$

and as $d \widehat{\Upsilon}: \mathcal{W}_{\widehat{x}_{t, s}} \rightarrow T_{\widehat{w}} \widehat{W}$ is an isomorphism, we conclude that $\left.\frac{d}{d s}\right|_{s=0} \vec{Y}_{t, s}^{\mathcal{W}}=0$.

The map $d \Phi_{s}^{\vec{X}}$ induces an isometry on $T \widehat{\mathcal{F}}^{\perp}$, so the length of the vectors $\vec{Y}_{t, s}^{\widehat{\Upsilon}}$ is a constant function of $s$. At $s=0$ we have that $\vec{Y}_{t, 0}^{\widehat{\Upsilon}}=\widehat{H}^{\prime}(\widehat{x}, t)^{\widehat{\Upsilon}}=0$, as $\widehat{H}^{\prime}(\widehat{x}, t) \in \mathcal{W}_{\widehat{x}_{t}}$. Hence, $\vec{Y}_{t, s}^{\widehat{\Upsilon}}=0$ for all $s$. Thus, we have

$$
\begin{aligned}
L_{\vec{X}} H^{\prime}(\widehat{x}, t) & =\left.\frac{d}{d s}\right|_{s=0} \vec{Y}_{t, s}=\left.\frac{d}{d s}\right|_{s=0} \vec{Y}_{t, s}^{\widehat{\mathcal{F}}}+\left.\frac{d}{d s}\right|_{s=0} \vec{Y}_{t, s}^{\widehat{\Upsilon}}+\left.\frac{d}{d s}\right|_{s=0} \vec{Y}_{t, s}^{\mathcal{W}} \\
& =\left.\frac{d}{d s}\right|_{s=0} \vec{Y}_{t, s}^{\widehat{\mathcal{F}}} \in T_{\widehat{x_{t}}} \widehat{\mathcal{F}} .
\end{aligned}
$$

This completes the proof of Proposition 5.1

Corollary 5.2. Let $\mathcal{F}$ be a Riemannian foliation of a compact manifold $M$; then

$$
\operatorname{cat}_{\mathbf{O}(q)}(\widehat{M}, \widehat{\mathcal{F}})=\operatorname{cat}_{\mathbf{O}(q)}(\widehat{W}) .
$$

Note that the proof of Proposition 5.1 implies that

$$
\operatorname{cat}_{\mathbf{O}(q)}(\widehat{M}, \widehat{\mathcal{F}})=\operatorname{cat}_{\mathbf{O}(q)}(\widehat{M}, \widehat{\mathcal{E}}) .
$$

This is equivalent to (29) as there is a natural equivalence

$$
\operatorname{cat}_{\mathbf{O}(q)}(\widehat{M}, \widehat{\mathcal{E}})=\operatorname{cat}_{\mathbf{O}(q)}(\widehat{W}),
$$

since $\widehat{\Upsilon}: \widehat{M} \rightarrow \widehat{W}$ is a fibration with compact fibers.

\section{6. $G$-EQUivariant CATEGORY AND ORBIT TYPE}

In this section, we recall some general properties of a smooth action of a compact Lie group $G$ on a compact manifold and their applications to $G$-equivariant category. References for this material are [3, 4, 7, 14, 21, 46, 51, 68. These results will be applied to the case of the $\mathbf{O}(q)$-action on $\widehat{W}$ in the next section.

Let $G$ be a compact Lie group, and $R: N \times G \rightarrow N$ a smooth right action on a closed manifold $N$ such that the quotient $N / G$ is connected. For $A \in G$, denote $u A=R(u, A)$, and let $u G=\{u A \mid A \in G\}$ denote the orbit of $u$. Define the stabilizer subgroup of the action of $G$ on $N$,

$$
G_{0}=\{A \in G \mid u A=u \text { for all } u \in N\} .
$$


Note that $G_{0}$ is always closed and normal. The action of $G$ is said to be effective if $G_{0}$ is the trivial subgroup.

Let $U \subset N$ be a $G$-invariant open set. A map $\mathcal{H}: U \times[0,1] \rightarrow N$ is said to be a $G$-homotopy if $H$ is $G$-equivariant. It is $G$-categorical if, in addition, $\mathcal{H}_{0}: U \rightarrow N$ is the inclusion and $\mathcal{H}_{1}: U \rightarrow u G$ has image in a single orbit, for some $u \in N$. A $G$-invariant subset $U \subset N$ is $G$-categorical if there exists a $G$-categorical homotopy $\mathcal{H}: U \times[0,1] \rightarrow N$. The $G$-category $\operatorname{cat}_{G}(N)$ of $N$ is the least number of $G$ categorical open sets required to cover $N$.

We recall some basic aspects of the geometry of a smooth $G$-action. We assume that $N$ has a $G$-invariant Riemannian metric $g_{N}$ on $T N$. For $u \in N, w \in u G$, and $\epsilon>0$, define the $\epsilon$-normal bundles

$$
\begin{aligned}
\mathcal{N}(w, \epsilon) & =\left\{\vec{X} \in T_{w}(N) \mid \vec{X} \perp T_{w}(w G),\|\vec{X}\|<\epsilon\right\}, \\
\mathcal{N}(u G, \epsilon) & =\left\{\vec{X} \in T_{w}(N) \mid w \in u G, \vec{X} \perp T_{w}(u G),\|\vec{X}\|<\epsilon\right\} .
\end{aligned}
$$

Let exp : $T N \rightarrow N$ denote the geodesic exponential, and let $\exp _{w}: T_{w} N \rightarrow N$ for $w \in N$ be the exponential map based at $w$. The $\epsilon$-normal neighborhood to $u G$ is

$$
\mathcal{U}(u G, \epsilon)=\left\{\exp _{w}(\vec{X}) \mid w \in u G, \vec{X} \in \mathcal{N}(w, \epsilon)\right\} .
$$

For $w=u A, A \in G$, the derivative of the right action $R$ defines an isometric linear representation of the isotropy group,

$$
d_{w} R: G_{w} \rightarrow \operatorname{Isom}\left(T_{w}(u G)^{\perp}\right) .
$$

Note that for arbitrary $A \in G, d_{u} R_{A}: T_{u}(u G)^{\perp} \rightarrow T_{w}(u G)^{\perp}$ intertwines the representations $d_{w} R$ and $d_{u} R$.

Let $\mathcal{N}_{0}(w, \epsilon) \subset T_{w}(u G)^{\perp}$ denote the fixed vectors for the representation $d_{w} R$.

Theorem 6.1 (Equivariant Tubular Neighborhood). For $u \in N$, there exists $\epsilon>0$ so that the open neighborhood $U=\mathcal{U}(u G, \epsilon)$ is a strong $G$-equivariant deformation retract of $u G$. That is, there exists a G-equivariant homotopy $\mathcal{H}: U \times[0,1] \rightarrow N$ such that $\mathcal{H}_{t} \mid u G$ is the identity for all $0 \leq t \leq 1$, and $\mathcal{H}_{1}(U)=u G$. In particular, $U$ is a $G$-categorical open neighborhood of $u G$.

Proof. For $\epsilon>0$ sufficiently small, $\exp : \mathcal{N}(u G, \epsilon) \rightarrow N$ is a diffeomorphism. The homotopy $\mathcal{H}$ is then defined using the homothety

$$
\mathcal{H}\left(\exp _{w}(\vec{X}), t\right)=\exp _{w}(t \vec{X})
$$

of the normal geodesic map, where $\vec{X} \in \mathcal{N}(w, \epsilon)$.

The next result is a generalization of Theorem 6.1 See e.g. Theorem 4.8 in [45].

Theorem 6.2 (Generalized Tubular Neighborhood). Let $A \subset N$ be a $G$-invariant submanifold. Then there exists a $G$-invariant open neighborhood $A \subset U$ and a $G$ equivariant homotopy $\mathcal{H}: U \times[0,1] \rightarrow N$ such that $\mathcal{H}_{t} \mid A$ is the inclusion for all $0 \leq t \leq 1, \mathcal{H}_{0}$ is the inclusion and $\mathcal{H}_{1}(U)=A$.

If $H$ is a closed subgroup of $G$, we denote by $(H)$ the conjugacy class of $H$ in $G$. While for an orbit $u G$ the isotropy group $G_{v}=\{A \in G \mid v A=v\}$ depends on the choice of $v \in u G$, the conjugacy class $\left(G_{v}\right)$ does not, and is therefore an invariant of $u G$. The conjugacy class $\left(G_{u}\right)$ is called the orbit type of $u G$. 
We say that $u G$ is a principal orbit if $G_{u}=G_{0}$. An orbit $u G$ with dimension less than that of a principal orbit is said to be a singular orbit. If $u G$ has the same dimension as a principal orbit, but the inclusion $G_{0} \subset G_{u}$ is proper (and therefore with finite index), then $u G$ is said to be an exceptional orbit.

One of the basic results for a smooth action of a compact group on a compact manifold is that it has a finite set of orbit types, as a consequence of Theorem 6.1. (See Proposition 1.2, Chapter IV of [7, or Theorem 5.11 of [68]). Thus, for the action $R: N \times G \rightarrow N$ there exists a finite collection of closed subgroups $\left\{G_{0}, \ldots, G_{k}\right\}$ of $G$, where $G_{0}$ is defined by (30), such that for all $u \in N$, there exists $\ell$ such that $\left(G_{u}\right)=\left(G_{\ell}\right)$.

There is a partial order on the set of orbit types of the $G$-space $N$ : for $u, v \in N$,

$$
\left(G_{u}\right) \leq\left(G_{v}\right) \text { if } A G_{v} A^{-1} \subset G_{u} \text { for some } A \in G .
$$

We adopt the notation $[u]=\left(G_{u}\right)$. Then $[u] \leq[v]$ (resp. $[u]<[v]$ ) means that the isotropy group of $v$ is conjugate to (resp. is a proper of) a subgroup of the isotropy group of $u$. Thus, the orbit $v G \cong G_{v} \backslash G$ is a fibration over the orbit $u G \cong G_{u} \backslash G$ and therefore should be considered "larger". Note that $[u] \leq\left(G_{0}\right)$ for all $u \in N$.

Given a closed subgroup $H$ of $G$, define the $(H)$-orbit type subspaces

$$
\begin{aligned}
N_{(H)} & =\left\{u \in N \mid\left(G_{u}\right)=(H)\right\}, \\
N_{\leq(H)} & =\left\{u \in N \mid\left(G_{u}\right) \leq(H)\right\},
\end{aligned}
$$

where $N_{(H)}$ is non-empty if and only if $H=G_{\ell}$ some $0 \leq \ell \leq k$; define $N_{\ell}=N_{\left(G_{\ell}\right)}$.

Here are some standard properties of the orbit type spaces; see [7, 21, 68] for details.

Theorem 6.3. For $0 \leq \ell \leq k, N_{\ell}$ is a G-invariant submanifold, the quotient space $N_{\ell} / G$ is a smooth manifold, and the quotient map

$$
\pi_{\ell}: N_{\ell} \longrightarrow N_{\ell} / G
$$

is a fibration. Moreover, $\pi_{\ell}$ has the $G$-equivariant path lifting property: given a smooth path $\sigma:[0,1] \rightarrow N_{\ell} / G$ with $\sigma(0)=v$, there exists a $G$-equivariant smooth map $\Sigma: v G \times[0,1] \rightarrow N_{\ell}$ such that $\Sigma_{0}: v \cdot G \rightarrow N_{\ell}$ is the inclusion of the orbit $v G$, and $\pi_{\ell} \circ \Sigma(v A, t)=\sigma(t)$ for all $A \in G$.

Corollary 6.4. Given a smooth path $\sigma:[0,1] \rightarrow N_{\ell}$ with $v=\sigma(0)$, there exists a G-equivariant map $\Sigma: v G \times[0,1] \rightarrow N_{\ell}$, such that $\Sigma_{0}$ is the inclusion and $\pi_{\ell} \circ$ $\Sigma(v A, t)=\pi_{\ell} \circ \sigma(t)$.

There are various subtleties which arise in the study of the orbit-type spaces $N_{(H)}$ for a smooth compact Lie group action. One is that the quotient space $N_{\ell} / G$ need not be connected, and the connected components of $N_{\ell} / G$ need not all have the same dimensions. A second issue is that $N_{(H)}$ need not be closed, and the structure of the closure $\overline{N_{(H)}}$ is a fundamental aspect of the study of the action. We consider both of these points in the following.

Proposition 6.5. Assume $N / G$ is connected. Then the principal orbit space $N_{0}=$ $N_{\left(G_{0}\right)}$ is an open dense $G$-invariant submanifold of $N$ such that $N_{0} / G$ is connected. For $1 \leq \ell \leq k$, the submanifold $N_{\ell} \subset N$ has codimension at least two. If there are no exceptional orbit types, then $N_{0}$ is connected. 
For example, see Theorem 5.14 and Proposition 5.15 of 68. The case where $N_{0}$ is not connected occurs when there exists $A \in G$ that acts as an involution of $N$ with a codimension-one fixed-point set.

Next, we introduce the $\mathcal{Z}$-stratification of $N$ associated to the orbit-type decomposition. For $u \in N$, let $\mathcal{Z}_{u} \subset N$ denote the $G$-orbit of the connected component of $N_{\left(G_{u}\right)}$ containing $u$. Note that for $u, v \in N$, either $\mathcal{Z}_{u} \cap \mathcal{Z}_{v}=\emptyset$ or $\mathcal{Z}_{u}=\mathcal{Z}_{v}$. Note that if $G$ is connected, then $\mathcal{Z}_{u}$ is also connected. The inclusion $u G \subset \mathcal{Z}_{u}$ can be strict. For example, this is always the case when $\mathcal{Z}_{u}$ is not a closed subset of $N$.

Proposition 6.6. The collection of sets $\mathcal{Z}_{u}$ for $u \in N$ form a finite stratification of $N$. That is, there exists a finite set of points $\left\{\eta_{0}, \ldots, \eta_{K}\right\} \subset N$ such that for $\mathcal{Z}_{i}=\mathcal{Z}_{\eta_{i}}, \mathcal{Z}_{0}=N_{0}$ and

$$
N=\mathcal{Z}_{0} \cup \cdots \cup \mathcal{Z}_{K}
$$

Proof. There exists a finite number of orbit types, and for each orbit type $\left(G_{\ell}\right)$ the space $N_{\left(G_{\ell}\right)}$ is a finite union of connected submanifolds.

The $\mathcal{Z}$-stratification of $N$ is the collection of sets

$$
\mathfrak{M}_{G}(N)=\left\{\mathcal{Z}_{0}, \mathcal{Z}_{1}, \ldots, \mathcal{Z}_{K}\right\} .
$$

It satisfies the axioms of a Whitney stratification (see Chapter 2 of [21]).

For the study of $G$-category, it is more natural to consider the $\mathcal{Z}$-stratification of $N$ than the orbit-type stratification by the manifolds $N_{\ell}$. We will see that the $\mathcal{Z}$-stratification captures more of the $G$-homotopical information about the action.

A fundamental property of the $\mathcal{Z}$-stratification is the incident relations between the closures $\overline{\mathcal{Z}_{\ell}}$ of the strata. This motivates the following definition, which we will subsequently relate to the order-type relations between the strata. Define the incidence partial order on the collection of sets $\left\{\mathcal{Z}_{1}, \ldots, \mathcal{Z}_{K}\right\}$ by setting

$$
\mathcal{Z}_{i} \lesssim \mathcal{Z}_{j} \Longleftrightarrow \mathcal{Z}_{i} \subset \overline{\mathcal{Z}_{j}}
$$

Note that $\mathcal{Z}_{i} \lesssim \mathcal{Z}_{j}$ and $\mathcal{Z}_{j} \lesssim \mathcal{Z}_{i}$ implies that $\mathcal{Z}_{i}=\mathcal{Z}_{j}$.

We require two fundamental technical lemmas, used to study the properties of the incidence partial order. The first implies that the function $u \mapsto[u]$ is "lower semicontinuous" on $N$.

Lemma 6.7. For $u \in N$ and $\epsilon>0$ sufficiently small, let $\mathcal{U}(u G, \epsilon)$ be an $\epsilon$-normal neighborhood. Then $[u] \leq[v]$ for all $v \in \mathcal{U}(u G, \epsilon)$. Moreover, $v \in \mathcal{U}(u G, \epsilon)$ satisfies $[u]=[v]$ if and only if $v=\exp _{w}(\vec{X})$ for some $w \in u G$ and $\vec{X} \in \mathcal{N}_{0}(w, \epsilon)$.

Proof. Recall from Theorem 6.1 that for $v \in \mathcal{U}(u G, \epsilon)$ there exists $w \in u A$ and $\vec{X} \in \mathcal{N}(w, \epsilon)$ such that $v=\exp _{w}(\vec{X})$. Then $B \in G_{v}$ if and only if $w=w B$ and $d_{w} R_{B}(\vec{X})=\vec{X}$. Let $A \in G$ such that $w=u A$; then $B \in A^{-1} G_{u} A$, so $G_{v} \subset A^{-1} G_{u} A$ and hence $[u] \leq[v]$. If $[u]=[v]$, then for all $B \in A^{-1} G_{u} A$ we have that $d_{w} R_{B}(\vec{X})=\vec{X}$, hence $\vec{X} \in \mathcal{N}_{0}(w, \epsilon)$. Conversely, $\vec{X} \in \mathcal{N}_{0}(w, \epsilon)$ implies $G_{v}=A^{-1} G_{u} A$, hence $[u]=[v]$.

The next result implies that the function $t \mapsto[\mathcal{H}(u, t)]$ is "upper semicontinuous" for $G$-equivariant homotopy.

Lemma 6.8. Let $\mathcal{H}: U \times[0,1] \rightarrow N$ be a G-homotopy. For $u \in U$, set $u_{t}=\mathcal{H}(x, t)$. Then for $0 \leq t \leq 1$, we have $G_{u} \subset G_{u_{t}}$ and therefore $\left[u_{t}\right] \leq[u]$.

Proof. For $A \in G_{u}$ then $u_{t} A=\mathcal{H}(u, t) A=\mathcal{H}(u A, t)=\mathcal{H}(u, t)=u_{t}$. 
The following result establishes the relationships between the incidence partial order and the orbit-type partial order.

Proposition 6.9. Let $v \in \mathcal{Z}_{i}$ and $u \in \mathcal{Z}_{j}$, for $i \neq j$ (and hence $\mathcal{Z}_{i} \cap \mathcal{Z}_{j}=\emptyset$ ).

Suppose that $v \in \overline{\mathcal{Z}_{j}}-\mathcal{Z}_{j}$. Then $[v]<[u]$ and $\mathcal{Z}_{i} \subset\left(\overline{\mathcal{Z}_{j}}-\mathcal{Z}_{j}\right)$, hence $\mathcal{Z}_{i} \lesssim \mathcal{Z}_{j}$. In particular, $\mathcal{Z}_{i} \lesssim \mathcal{Z}_{j}$, and $\mathcal{Z}_{i} \neq \mathcal{Z}_{j}$ implies that $[v]<[u]$.

Proof. Given that $v \in \overline{\mathcal{Z}_{j}}-\mathcal{Z}_{i}$ there exists a sequence $\left\{u_{\ell} \mid \ell=1,2, \ldots\right\} \subset \mathcal{Z}_{j}$ such that $\lim _{\ell \rightarrow \infty} u_{\ell}=v$. Let $\epsilon>0$ be such that $\mathcal{U}(v G, \epsilon)$ is a $G$-categorical neighborhood of $v G$. Then there exists $\ell$ such that $u_{\ell} \in \mathcal{U}(v G, \epsilon)$. Let $w \in v G, \vec{X} \in \mathcal{N}(w, \epsilon)$ be such that $\exp _{w}(\vec{X})=u_{\ell}$. Then by Lemma 6.7. $[v]<\left[u_{\ell}\right]=[u]$ unless $\vec{X} \in \mathcal{N}_{0}(w, \epsilon)$. If $\vec{X} \in \mathcal{N}_{0}(w, \epsilon)$, we have that $[v]=\left[u_{\ell}\right]=[u]$, and hence $\exp _{w}\left(\mathcal{N}_{0}(w, \epsilon)\right) \subset \mathcal{Z}_{j}$. Thus, $v \in \mathcal{Z}_{j}$, contrary to our assumption, so we must have $[v]<[u]$.

It remains to show that $\mathcal{Z}_{i} \cap\left(\overline{\mathcal{Z}_{j}}-\mathcal{Z}_{j}\right) \neq \emptyset$ implies $\mathcal{Z}_{i} \subset\left(\overline{\mathcal{Z}_{j}}-\mathcal{Z}_{j}\right)$. This follows from an argument similar to the above.

Corollary 6.10. A stratum $\mathcal{Z}_{j}$ is minimal for the incidence partial order if and only if $\mathcal{Z}_{j}$ is a closed submanifold.

Proof. If $\mathcal{Z}_{j}$ is not closed, then there exists $v \in \overline{\mathcal{Z}_{j}}-\mathcal{Z}_{j}$, and then $v \in \mathcal{Z}_{i}$ for some $i \neq j$. Then $\mathcal{Z}_{i} \lesssim \mathcal{Z}_{j}$ by Proposition 6.9, so $\mathcal{Z}_{j}$ is not minimal. The converse is obvious.

The orbit-type function $u \rightarrow[u]$ is lower-semicontinuous by Lemma 6.7, so it is natural to also consider a notion of minimality based on continuity:

Definition 6.11. A stratum $\mathcal{Z}_{u}$ is said to be locally minimal if there is an open $G$-invariant neighborhood $U$ of the closure $\overline{\mathcal{Z}_{u}}$ such that $[u] \leq[v]$ for all $v \in U$.

Proposition 6.12. $\mathcal{Z}_{u}$ is locally minimal $\Leftrightarrow \mathcal{Z}_{u}$ is closed $\Leftrightarrow \mathcal{Z}_{u}$ is a least element for the incidence partial order.

Proof. Assume that $\mathcal{Z}_{u}$ is closed, so $\overline{\mathcal{Z}_{u}}=\mathcal{Z}_{u}$ is a submanifold. Then by the Generalized Tubular Neighborhood Theorem, Theorem 6.2, there exists a $G$-invariant open neighborhood $U$ of $\mathcal{Z}_{u}$ and a $G$-equivariant homotopy $\mathcal{H}: U \times[0,1] \rightarrow M$ such that $\mathcal{H}_{1}(U)=\mathcal{Z}_{u}$. We claim that for all $v \in U,[u] \leq[v]$. Let $v \in U$ and set $v_{t}=\mathcal{H}(v, t)$. Note that $v_{1} \in \mathcal{Z}_{u}$ so that $\left[v_{1}\right]=[u]$. By Lemma 6.8. $\left[v_{t}\right] \leq\left[v_{0}\right]=[v]$ for all $t$. In particular, $[u]=\left[v_{1}\right] \leq\left[v_{0}\right]=[v]$, as was to be shown.

Conversely, assume that $\mathcal{Z}_{u}$ is locally minimal, with a $G$-invariant open neighborhood $U$ of $\overline{\mathcal{Z}_{u}}$ as in the definition. If there exists $v \in \overline{\mathcal{Z}_{u}}-\mathcal{Z}_{u} \subset U$, then $[v]<[u]$ by Proposition 6.9. This contradicts the assumption that $v \in U$ satisfies $[v] \geq[u]$.

Corollary 6.13. For each $u \in N$, there exists a locally minimal stratum $\mathcal{Z}_{j}$ with $\mathcal{Z}_{j} \subset \overline{\mathcal{Z}_{u}}$.

Corollary 6.14. Let $\mathcal{Z}_{j}$ be a locally minimal stratum. Then there exists an index $\ell_{j}$ such that $\mathcal{Z}_{j}$ is a closed connected component of $\left(G_{\ell_{j}}\right)$.

For the remainder of this section, we consider the properties of $G$-homotopy with respect to the incidence and orbit-type partial orders, and give applications to $G$ category. The next result implies that $G$-homotopy preserves the $\mathcal{Z}$-stratification. 
Proposition 6.15. Let $\mathcal{H}: U \times[0,1] \rightarrow N$ be a $G$-homotopy with $G_{0}$ the inclusion. Suppose that $\mathcal{Z}_{u}$ is a locally minimal set for $u \in U$. Then for all $0 \leq t \leq 1$,

$$
\mathcal{H}_{t}\left(U \cap \mathcal{Z}_{u}\right)=\mathcal{Z}_{u} .
$$

Proof. For $u \in U$, set $u_{t}=\mathcal{H}(x, t)$. Then $u_{0}=u \in U \cap N_{\left(G_{u}\right)}$. Lemma 6.8 implies that for all $0 \leq t \leq 1,\left[u_{t}\right] \leq[u]$; hence $\left(G_{u_{t}}\right) \leq\left(G_{u}\right)$, so that $u_{t} \in N_{\leq\left(G_{u}\right)}$. It follows that a $G$-homotopy preserves connected components of the orbit-type filtration by the closed sets $\left\{N_{\leq\left(G_{\ell}\right)} \mid 0 \leq \ell \leq k\right\}$.

Now suppose that $u \in U \cap \mathcal{Z}_{u}$, where $\mathcal{Z}_{u}$ is locally minimal. Then by Corollary 6.10, the set $\mathcal{Z}_{u}$ is closed, hence is a connected component of $N_{\leq\left(G_{u}\right)}$. Thus $u_{t} \in \mathcal{Z}_{u}$ for all $0 \leq t \leq 1$.

As $\mathcal{Z}_{u}$ is a closed submanifold and $\mathcal{H}_{0}: \mathcal{Z}_{u} \rightarrow \mathcal{Z}_{u}$ is the identity, the map $\mathcal{H}_{t}$ must also be surjective for all $0 \leq t \leq 1$.

Next, we examine the relation between $G$-category and the $\mathcal{Z}$-stratification.

Proposition 6.16. Let $G$ be a compact Lie group, and $R: N \times G \rightarrow N$ a smooth right action on a closed manifold $N$ such that the quotient $N / G$ is connected. Then the $G$-category $\operatorname{cat}_{G}(N)$ is finite, and there exists a $G$-categorical covering $\left\{\mathcal{H}_{\ell}: U_{\ell} \times[0,1] \rightarrow N \mid 1 \leq \ell \leq \Lambda\right\}$ and basepoints $\left\{w_{1}, \ldots, w_{\Lambda}\right\} \subset N$ such that each $\mathcal{Z}_{w_{\ell}}$ is locally minimal with $H_{\ell}\left(U_{\ell}\right) \subset w_{\ell} G$.

Proof. By Lemma 6.7 every orbit has a $G$-categorical open neighborhood, and $N$ compact implies there is a finite subcovering by $G$-categorical open sets; hence $\operatorname{cat}_{G}(N)$ is finite. Set $\Lambda=\operatorname{cat}_{G}(N)$.

Let $\left\{\mathcal{H}_{\ell}^{\prime}: U_{\ell} \times[0,1] \rightarrow N \mid 1 \leq \ell \leq \Lambda\right\}$ be a $G$-categorical open covering, with $\mathcal{H}_{\ell}^{\prime}\left(U_{\ell}\right) \subset v_{\ell} G$ for points $\left\{v_{1}, \ldots, v_{k}\right\} \subset N$.

By Corollary 6.13, for each $1 \leq \ell \leq \Lambda$, we can choose $w_{\ell} \in \overline{Z_{v_{\ell}}}$ such that $\mathcal{Z}_{w_{\ell}}$ is a locally minimal set. Let $\mathcal{U}_{\ell}=\mathcal{U}\left(w_{\ell} G, \epsilon\right)$ be an $\epsilon$-normal neighborhood as in Theorem 6.1, with $G$-homotopy retract $\mathcal{H}^{\prime \prime \prime}: \mathcal{U}_{\ell} \times[0,1] \rightarrow N$. (We choose $\epsilon>0$ sufficiently small so that it works for all $\ell$.) Then there exists $u_{\ell} \in \mathcal{U}_{\ell} \cap Z_{v_{\ell}}$ such that $v_{\ell}$ and $u_{\ell}$ lie in the same path-component of the connected strata $Z_{v_{\ell}}$.

Let $\sigma_{\ell}:[0,1] \rightarrow Z_{v_{\ell}}$ be a smooth path such that $\sigma_{\ell}(0)=v_{\ell}$ and $\sigma_{\ell}(1)=u_{\ell}$. The path $\sigma_{\ell}$ defines a smooth $G$-equivariant lifting $\Sigma_{\ell}: v_{\ell} G \times[0,1] \rightarrow Z_{v_{\ell}}$ such that $\pi_{\ell} \circ \Sigma_{\ell}\left(v_{\ell} A, t\right)=\pi_{\ell} \circ \sigma_{\ell}(t)$ by Corollary 6.4. Thus, $\Sigma_{\ell}\left(v_{\ell} G, 1\right) \subset u_{\ell} G \subset \mathcal{U}_{\ell} \cap Z_{v_{\ell}}$.

Now, define $\mathcal{H}_{\ell}: U_{\ell} \times[0,1] \rightarrow N$ as the concatenation:

$$
\mathcal{H}_{\ell}(u, t)= \begin{cases}\mathcal{H}^{\prime}(u, 3 t) & \text { for } 0 \leq \leq t \leq 1 / 3, \\ \Sigma_{\ell}\left(\mathcal{H}_{\ell}^{\prime}(u, 1), 3 t-1\right) & \text { for } 1 / 3 \leq t \leq 2 / 3, \\ \mathcal{H}_{\ell}^{\prime \prime \prime}\left(\Sigma_{\ell}\left(\mathcal{H}_{\ell}^{\prime}(u, 1), 1\right), 3 t-2\right) & \text { for } 2 / 3 \leq t \leq 1 .\end{cases}
$$

This yields a piece-wise smooth $G$-categorical homotopy $\mathcal{H}$ as desired. By adjusting the time parameters, the map $\mathcal{H}_{\ell}$ is then modified to be smooth.

One of the main problems for the study of $G$-category is to obtain upper and lower bounds for $\operatorname{cat}_{G}(N)$ in terms of the geometry and topology of the $G$-action. We obtain two such estimates in terms of properties of the $\mathcal{Z}$-stratification.

Let $\alpha=\alpha_{G}(N)$ denote the number of locally minimum strata in $\mathfrak{M}_{G}(N)$. We can assume that the strata in $\mathfrak{M}_{G}(N)=\left\{\mathcal{Z}_{0}, \mathcal{Z}_{1}, \ldots, \mathcal{Z}_{K}\right\}$ are ordered such that $\mathcal{Z}_{\ell} \in \mathfrak{M}_{G}(N)$ is locally minimal exactly when $1 \leq j \leq \alpha$. (Recall that $\mathcal{Z}_{0}=N_{0}$ consists of regular orbits by previous assumption.) 
Theorem 6.17. $\operatorname{cat}_{G}(N) \geq \alpha_{G}(N)$. More precisely,

$$
\alpha_{G}(N) \leq \sum_{1 \leq j \leq \alpha} \operatorname{cat}_{G}\left(\mathcal{Z}_{j}\right) \leq \operatorname{cat}_{G}(N)
$$

Proof. Let $\mathcal{U}=\left\{\mathcal{H}_{\ell}: U_{\ell} \times[0,1] \rightarrow N \mid 1 \leq \ell \leq \Lambda\right\}$ be a $G$-categorical covering of $N$, and let $\left\{w_{1}, \ldots, w_{\Lambda}\right\} \subset N$ be such that $H_{\ell}\left(U_{\ell}\right) \subset w_{\ell} G$.

Let $\mathcal{Z}_{j}$ be a locally minimal set, and suppose that $u \in U_{\ell} \cap \mathcal{Z}_{j}$. Set $u_{\ell, t}=\mathcal{H}_{\ell}(u, t)$. Then by Proposition 6.15, $u_{\ell, t} \in \mathcal{Z}_{j}$ for all $0 \leq t \leq 1$, and hence $u_{\ell, 1} G=w_{\ell} G \subset \mathcal{Z}_{j}$.

Suppose that $\mathcal{Z}_{i}$ and $\mathcal{Z}_{j}$ are disjoint locally minimal sets, such that $u \in U_{\ell} \cap \mathcal{Z}_{i}$ and $v \in U_{k} \cap \mathcal{Z}_{j}$. Let $u_{\ell, t}=\mathcal{H}_{\ell}(u, t)$ with $w_{\ell}=u_{\ell, 1}$ and $v_{k, t}=\mathcal{H}_{k}(v, t)$ with $w_{k}=v_{k, 1}$. Then $w_{\ell} G \subset \mathcal{Z}_{i}$ and $w_{k} G \subset \mathcal{Z}_{j}$. If $k=\ell$, then $\mathcal{H}_{\ell}=\mathcal{H}_{k}$, and hence $w_{\ell}$ and $w_{k}$ lie in the same $G$-orbit. This implies that $\mathcal{Z}_{i} \cap \mathcal{Z}_{j} \neq \emptyset$, contrary to assumption. Thus, we must have that $\ell \neq k$.

For each $1 \leq j \leq \alpha$, define the subset of the $G$-categorical covering $\mathcal{U}$ by

$$
\mathcal{U}_{j}=\left\{\mathcal{H}_{\ell}: U_{\ell} \times[0,1] \rightarrow N \mid 1 \leq \ell \leq \Lambda \text { such that } \mathcal{Z}_{j} \cap U_{\ell} \neq \emptyset\right\} .
$$

Then by the above remarks, $\mathcal{U}_{i}$ and $\mathcal{U}_{j}$ are disjoint collections for $i \neq j$. Moreover, as $\mathcal{U}$ is an open covering of $N$, each $\mathcal{U}_{j}$ is non-empty.

Given $\ell$ with $\mathcal{Z}_{j} \subset U_{\ell}$, Proposition 6.15 implies that the restriction $U_{\ell} \mid \mathcal{Z}_{j}$ is a $G$-categorical open set for $\mathcal{Z}_{j}$. Hence, the restrictions to $\mathcal{Z}_{j}$ of the open sets in $\mathcal{U}_{j}$ define a $G$-categorical covering of $\mathcal{Z}_{j}$. It follows that the collection $\mathcal{U}_{j}$ must have at least $\operatorname{cat}_{G}\left(\mathcal{Z}_{j}\right)$ elements. The estimates in (39) now follow immediately.

There are also sophisticated lower bound estimates for $\operatorname{cat}_{G}(N)$ in terms of the cohomology and homotopy theory of the compact group action [19.

There are also several types of upper bound estimates for cat $G(N)$ as discussed for example in the works of Marzantowicz [46, 51], Bartsch [4, Ayala, Lasheras and Quintero [3], Colman [14] and the authors [38. The simplest version is based on the dimension estimate that for a connected manifold $X$, there is an upper bound $\operatorname{cat}(X) \leq \operatorname{dim}(X)+1$. The quotient $\mathcal{Z}_{\ell} / G$ is a connected manifold for each stratum $\mathcal{Z}_{\ell} \in \mathfrak{M}_{G}(N)=\left\{\mathcal{Z}_{0}, \mathcal{Z}_{1}, \ldots, \mathcal{Z}_{K}\right\}$, hence cat ${ }_{G}\left(\mathcal{Z}_{\ell}\right) \leq \operatorname{dim}\left(\mathcal{Z}_{\ell} / G\right)+1$. Combine this with the previous remarks and the estimate (39) to obtain the general estimate

$$
\sum_{1 \leq j \leq \alpha_{G}(N)} \operatorname{cat}_{G}\left(\mathcal{Z}_{j}\right) \leq \operatorname{cat}_{G}(N) \leq \sum_{0 \leq \ell \leq K}\left\{\operatorname{dim}\left(\mathcal{Z}_{\ell} / G\right)+1\right\} .
$$

\section{IsOtopy AND $\mathcal{Z}$-STRATIFICATIONS FOR $\mathcal{F}$}

In this section, the results of the last section are applied to the $\mathbf{O}(q)$-manifold $\widehat{M}$ associated to a Riemannian foliation $\mathcal{F}$ of a closed manifold $M$. We introduce the isotopy stratification of $M$, corresponding to the structure of the leaves of $\mathcal{F}$. The main result is that the $\mathcal{Z}$-stratification of $\widehat{W}$ corresponds to the isotopy stratification of $M$. This yields an interpretation of the locally minimal sets $\mathcal{Z}_{u}$ for the $\mathbf{O}(q)$-action on $\widehat{M}$ in terms of the intrinsic geometry of $\mathcal{F}$. References for this section are the works of Molino [55, 56, 57, Haefliger [30, 31] and Salem 63].

Let $L$ be a leaf of $\mathcal{F}$. An $\mathcal{F}$-isotopy of $L$ is a smooth map $I: L \times[0,1] \rightarrow M$ such that $I_{0}: L \rightarrow M$ is the inclusion of $L$, and for each $0 \leq t \leq 1, I_{t}: L \rightarrow M$ is a diffeomorphism onto a leaf $L_{t}$ of $\mathcal{F}$. We then say that the leaf $L_{1}$ is $\mathcal{F}$-isotopic to $L_{0}=L$, and write $L_{0} \sim L_{1}$. For example, Proposition 3.1 implies that every leaf

of $\widehat{\mathcal{F}}$ is $\widehat{\mathcal{F}}$-isotopic in $\widehat{M}$ to every other leaf of $\widehat{\mathcal{F}}$. For $\mathcal{F}$ this need not be true. 
Let $\mathcal{I}_{L}$ denote the set of leaves of $\mathcal{F}$ which are $\mathcal{F}$-isotopic to $L$. For $L=L_{x}$ set $\mathcal{I}_{x}=\mathcal{I}_{L_{x}}$. The collection of $\mathcal{F}$-isotopy classes of the leaves of $\mathcal{F}$ defines the isotopy stratification of $M$.

Next, we use the Molino theory to define the holonomy stratification for $M$, which is derived from the $\mathcal{Z}$-stratification of $\widehat{W}$ introduced in the last section.

For $\widehat{x}=(x, e) \in \widehat{M}$, set $\widehat{w}=\widehat{\Upsilon}(\widehat{x})$; then the fiber $\widehat{\Upsilon}^{-1}(w)=\bar{L}_{\widehat{x}}$. The $\mathbf{O}(q)$-orbit of $\widehat{w}$ lifts to the $\mathbf{O}(q)$-orbit of $\bar{L}_{\widehat{x}}$, which projects to the leaf closure $\bar{L}_{x}$. Thus, each orbit $\widehat{w} \mathbf{O}(q)$ corresponds to exactly one leaf closure $\bar{L}_{x}$ of $\mathcal{F}$.

Recall that $\mathcal{E}$ denotes the singular Riemannian foliation of $M$ defined by the closures of the leaves of $\mathcal{F}$. Let $\Upsilon: M \rightarrow W$ denote the quotient map to the leaf space of $\mathcal{E}$. Then we have a commutative diagram of $\mathbf{O}(q)$-equivariant maps:

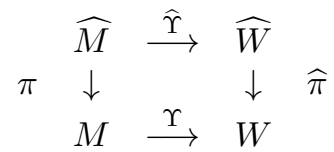

The projection $\pi$ restricts to a covering map $\pi: \widehat{L}_{\widehat{x}} \rightarrow L_{x}$, and by Corollary 3.9

$$
\bar{L}_{x}=\pi\left(\bar{L}_{\widehat{x}}\right) \cong \bar{L}_{\widehat{x}} / \mathbf{H}_{\widehat{x}},
$$

where $\mathbf{H}_{\widehat{x}} \equiv\left\{A \in \mathbf{O}(q) \mid \bar{L}_{\widehat{x}} A=\bar{L}_{\widehat{x}}\right\}=\overline{\mathcal{H}_{\widehat{x}}}$ by (12), and thus $\mathbf{H}_{\widehat{x}}=\mathbf{O}(q)_{\widehat{w}}$. It is immediate from the definitions that $\widehat{\Upsilon}(\widehat{y}) \in \widehat{W}_{\mathbf{O}}(q)_{\widehat{w}}$ if and only if $\mathcal{H}_{\widehat{y}}$ is conjugate in $\mathbf{O}(q)$ to a dense subgroup of $\mathbf{H}_{\widehat{x}}$.

Given a closed subgroup $H \subset \mathbf{O}(q), \widehat{x}=(x, e) \in \widehat{M}$ and $\widehat{w}=\widehat{\Upsilon}(\widehat{x})$, set

$$
\widehat{M}_{(H)}=\widehat{\Upsilon}^{-1}\left(\widehat{W}_{(H)}\right), \widehat{M}_{\leq(H)}=\widehat{\Upsilon}^{-1}\left(\widehat{W}_{\leq(H)}\right), \widehat{Z}_{\widehat{x}}=\widehat{\Upsilon}^{-1}\left(\mathcal{Z}_{\widehat{w}}\right)
$$

and project these $\mathbf{O}(q)$-invariant sets to $M$ to obtain

$$
M_{(H)}=\pi\left(\widehat{M}_{(H)}\right), M_{\leq(H)}=\pi\left(\widehat{M}_{\leq(H)}\right), Z_{x}=\pi\left(\widehat{Z}_{\widehat{x}}\right) .
$$

Note that as $\widehat{\Upsilon}$ has connected fibers, $\widehat{Z}_{\widehat{x}}$ can also be described as the $\mathbf{O}(q)$ orbit of the connected component of $\widehat{M}_{\left(\mathbf{O}(q)_{\hat{x}}\right)}$ containing $\widehat{x}$, and $Z_{x}$ is the connected component of $M_{\left(\mathbf{O}(q)_{x}\right)}$ containing $x$.

For a Riemannian foliation, the set of leaves of $\mathcal{F}$ without holonomy form an open dense subset, $M_{0} \subset M$. A leaf $L \subset M_{0}$ is said to be regular. Define open dense subsets $\widehat{M}_{0}=\pi^{-1}\left(M_{0}\right)$ and $\widehat{W}_{0}=\widehat{\Upsilon}\left(\widehat{M}_{0}\right) \subset \widehat{W}$. The leaves in $\widehat{M}_{0}$ are also said to be regular, and the points in the projection $\widehat{W}_{0}$ are regular orbits.

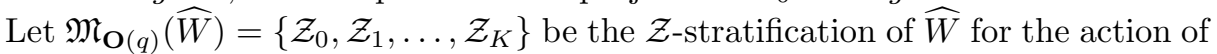
$\mathbf{O}(q)$. Define $Z_{j}=\pi\left(\widehat{\Upsilon}^{-1}\left(\mathcal{Z}_{j}\right)\right)$. Then the collection $\mathfrak{M}_{\mathcal{F}}(M)=\left\{Z_{0}, Z_{1}, \ldots, Z_{K}\right\}$ defines a Whitney stratification of $M$.

A stratum $Z_{j}$ is said to be locally minimal if the set $\mathcal{Z}_{j}$ is locally minimal. Let $\alpha=\alpha_{\mathcal{F}}(M)$ denote the number of locally minimum strata in $\mathfrak{M}_{\mathcal{F}}(M)$, and as before, we assume that the strata in $\mathfrak{M}_{\mathcal{F}}(M)$ are ordered such that $Z_{j}$ is locally minimal exactly when $1 \leq j \leq \alpha$.

Proposition 7.1. If $\widehat{y} \in \widehat{Z}_{\widehat{x}}$, then $\mathcal{H}_{\widehat{y}}$ is conjugate to $\mathcal{H}_{\widehat{x}}$ in $\mathbf{O}(q)$.

Proof. Let $\widehat{x}=(x, e)$. Given $\widehat{y}=(y, f) \in \widehat{Z}_{\widehat{x}}$, there exists $A \in \mathbf{O}(q)$ such that $\widehat{x}$ and $\widehat{y} A=(y, f A)$ lie in the same path component. As we must prove that $\mathcal{H}_{\widehat{y}}$ is 
conjugate to $\mathcal{H}_{\widehat{x}}$ in $\mathbf{O}(q)$, without loss we can assume that $A=I d$; hence there exists a continuous path $\sigma:[0,1] \rightarrow \widehat{M}_{\left(\mathbf{O}(q)_{\widehat{x}}\right)}$ such that $\sigma(0)=\widehat{x}$ and $\sigma(1)=\widehat{y}$.

For each $\widehat{y}_{t}=\sigma(t)$, by Corollary 3.5 there exists a linear model for $\mathcal{F}$ along the leaf $L_{y_{t}}$ through the point $y_{t}=\pi\left(\widehat{y}_{t}\right)$. The image $\pi(\sigma[0,1])$ is compact, and so is covered by a finite collection of such linear models. Thus, it suffices to consider the case where there is a foliated immersion as given in Corollary 3.5

$$
\Xi_{\widehat{x}}: Q^{\epsilon} \mid L_{x}=\left\{\left(\widehat{L}_{\widehat{x}} \times \mathbb{D}_{\epsilon}^{q}\right) / \pi_{1}\left(L_{x}, x\right)\right\} \longrightarrow M,
$$

and $y \in Z_{x}$ such that $y \in \mathcal{T}_{x}^{\epsilon} \cap Z_{x}$. Recall from (17) that for $\vec{X} \in \mathbb{D}_{\epsilon}^{q}$, we defined

$$
\iota_{\widehat{x}}(\vec{X})=\Xi_{\widehat{x}}(\xi), \text { where } \xi=\{(\{\widehat{x}\} \times\{\vec{X}\}) / \sim\} \in Q^{\epsilon} \mid L_{x} \text {, and set } \mathcal{T}_{x}^{\epsilon}=\iota_{\widehat{x}}\left(\mathbb{D}_{\epsilon}^{q}\right) .
$$

The map $\Xi_{\widehat{x}}$ defines an orthonormal framing of $Q_{y}$ which we denote by $f^{\prime}$, so that $\widehat{y}^{\prime}=\left(y, f^{\prime}\right) \in \widehat{M}$. Note that $f^{\prime}=f A^{\prime}$ for some $A^{\prime} \in \mathbf{O}(q)$ as $\pi(\widehat{y})=\pi\left(\widehat{y}^{\prime}\right)$. Thus, without loss we can assume that $\widehat{y}=\widehat{y}^{\prime}$.

By Lemma 3.2 and Proposition 3.4 the holonomy group $\mathcal{H}_{\xi}^{\omega}$ at the point $\xi$ of the foliation $\mathcal{F}^{\omega}$ on $Q^{\epsilon} \mid L_{x}$ is given by

$$
\mathcal{H}_{\xi}^{\omega}=\left\{A \in \mathcal{H}_{\widehat{x}} \mid \vec{X} A=\vec{X}\right\} \subset \mathcal{H}_{\widehat{x}} \subset \mathbf{O}(q) .
$$

As $\mathcal{F}^{\omega}$ is the pull-back of $\mathcal{F}$ via the map $\Xi_{\widehat{x}}$, it follows that $\mathcal{H}_{\xi}^{\omega}$ is conjugate to $\mathcal{H}_{\widehat{y}}$.

Let $\mathcal{V}_{\widehat{x}} \subset \mathbb{D}_{\epsilon}^{q}$ be the linear subspace of vectors fixed by the subgroup $\mathcal{H}_{\widehat{x}}$. Note that $\mathcal{V}_{\widehat{x}}$ is also the set of vectors fixed by the closure $\mathbf{H}_{\widehat{x}}=\overline{\mathcal{H}_{\widehat{x}}} \subset \mathbf{O}(q)$.

Similarly, define $\mathcal{V}_{\xi} \subset \mathbb{D}_{\epsilon}^{q}$ as the linear subspace of vectors fixed by $\mathcal{H}_{\xi}^{\omega}$. Again, $\mathcal{V}_{\xi}$ is also the set of vectors fixed by the closure $\overline{\mathcal{H}_{\xi}^{\omega}} \subset \mathbf{O}(q)$ which is conjugate to $\mathbf{H}_{\widehat{y}}$.

The key to the proof of Proposition 7.1 is the following result for linear actions.

Lemma 7.2. $\mathcal{H}_{\xi}^{\omega}=\mathcal{H}_{\widehat{x}} \Longleftrightarrow \mathcal{V}_{\xi}=\mathcal{V}_{\widehat{x}} \Longleftrightarrow\left(\mathbf{H}_{\widehat{y}}\right)=\left(\mathbf{H}_{\widehat{x}}\right)$.

Proof. Note that we always have $\mathcal{H}_{\xi}^{\omega} \subset \mathcal{H}_{\widehat{x}}$; hence $\overline{\mathcal{H}_{\xi}^{\omega}} \subset \mathbf{H}_{\widehat{x}}$ and so $\mathcal{V}_{\widehat{x}} \subset \mathcal{V}_{\xi}$.

Given that $\mathcal{H}_{\xi}^{\omega}=\mathcal{H}_{\widehat{x}}$, the other two equalities follow immediately.

Given that $\mathcal{V}_{\xi}=\mathcal{V}_{\widehat{x}}$, by (46) the vector $\vec{X} \in \mathcal{V}_{\widehat{x}}$, hence $\mathcal{H}_{\xi}^{\omega}=\mathcal{H}_{\widehat{x}}$.

Since $\mathcal{V}_{\widehat{x}}$ and $\mathcal{V}_{\xi}$ are closed subspaces, saying $\mathcal{V}_{\widehat{x}} \subset \mathcal{V}_{\xi}$ is proper implies that the inclusion $\mathbf{H}_{\widehat{x}} \subset \overline{\mathcal{H}_{\xi}^{\omega}}$ is proper. Hence, $\mathbf{H}_{\widehat{x}}=\overline{\mathcal{H}_{\xi}^{\omega}}$ implies that $\mathcal{V}_{\xi}=\mathcal{V}_{\widehat{x}}$.

We conclude the proof of Proposition 7.1. Given that $\widehat{y} \in \widehat{Z}_{\widehat{x}}$ we have $\left(\mathbf{H}_{\widehat{y}}\right)=$ $\left(\mathbf{H}_{\widehat{x}}\right)$. By Lemma 7.2 we have that $\mathcal{H}_{\xi}^{\omega}=\mathcal{H}_{\widehat{x}}$, thus $\mathcal{H}_{\xi}^{\omega}$ is conjugate to $\mathcal{H}_{\widehat{x}}$.

Proposition 7.3. For all $x \in M, Z_{x}=\mathcal{I}_{x}$. Hence, the isotopy stratification of $M$ is finite.

Proof. Let $\widehat{x}=(x, e) \in \widehat{M}$; then there is a local linear model given by (45). As in the proof of Proposition [7.1] it will suffice to show that $Z_{x} \cap \mathcal{T}_{x}^{\epsilon}=\mathcal{I}_{x} \cap \mathcal{T}_{x}^{\epsilon}$.

We first show that $Z_{x} \cap \mathcal{T}_{x}^{\epsilon} \subset \mathcal{I}_{x} \cap \mathcal{T}_{x}^{\epsilon}$.

Let $y \in Z_{x} \cap \mathcal{T}_{x}^{\epsilon}$, and let $\vec{X} \in \mathbb{D}_{\epsilon}^{q}$ so that $\iota_{\widehat{x}}(\vec{X})=\Xi_{\widehat{x}}(\xi)=y$, where $\xi \in Q^{\epsilon} \mid L_{x}$. $\widehat{M}$.

Let $f$ denote the orthonormal framing of $Q_{y}$ defined by $\Xi_{\widehat{x}}$, so that $\widehat{y}=(y, f) \in$ 
Then by Proposition [7.1 we have that $\mathcal{H}_{\xi}^{\omega}=\mathcal{H}_{\widehat{x}}$, hence $\vec{X} \in \mathcal{V}_{\widehat{x}}$ by the proof of Lemma 7.2. Define an isotopy $I: L_{x} \times[0,1] \rightarrow M$ where for $\widehat{z} \in L_{\widehat{x}}$ with $\pi(\widehat{z})=z$,

$$
I(z, t)=\Xi_{\widehat{x}}(\{\widehat{z}\} \times\{t \vec{X}\}),
$$

where the map is well-defined as $\vec{X} \in \mathcal{V}_{\widehat{x}}$ and the action of $\pi_{1}\left(L_{x}, x\right)$ on $\mathcal{V}_{\widehat{x}}$ is trivial. For the same reason, for all $0 \leq t \leq 1$, the restriction $I_{t}: L_{x} \rightarrow M$ is a diffeomorphism onto the leaf through $\iota_{\widehat{x}}(t \vec{X})$. As $\iota_{\widehat{x}}(\vec{X})=y$ and $\iota_{\widehat{x}}(0)=x$, this proves that $y \in \mathcal{I}_{x} \cap \mathcal{T}_{x}^{\epsilon}$.

Conversely, to show that $\mathcal{I}_{x} \cap \mathcal{T}_{x}^{\epsilon} \subset Z_{x} \cap \mathcal{T}_{x}^{\epsilon}$, let

$$
I: L_{x} \times[0,1] \rightarrow M \text { with } I_{0}(x)=x, I_{1}(x)=y
$$

be a foliated isotopy with $I_{t}(x) \in \mathcal{T}_{x}^{\epsilon}$ for all $0 \leq t \leq 1$. We must show that $L_{t} \subset Z_{x}$ for all $0 \leq t \leq 1$. It suffices to show that the conjugacy class of the closure $\left(\mathbf{H}_{\widehat{x}_{t}}\right)$ is constant. We are given the fact that the image $L_{t}=I_{t}\left(L_{x}\right)$ is isotopic to $L_{x}$. Hence the local covering maps $L_{t} \rightarrow L_{x}$ induced by (45) are diffeomorphisms; thus the holonomy groups $\mathcal{H}_{\widehat{x}_{t}}$ have constant conjugacy classes.

Corollary 7.4. For $\widehat{x}=(x, e) \in \widehat{M}$, set $\widehat{w}=\widehat{\Upsilon}(\widehat{x})$. Then $\mathcal{Z}_{\widehat{w}}$ is locally minimal if and only if $\mathcal{I}_{x}$ is a closed submanifold of $M$.

Corollary 7.5. Given the fact $x \in M$, let $\left(\mathcal{H}_{x}\right)$ denote the conjugacy class in $\mathbf{O}(q)$ of the holonomy group $\mathcal{H}_{\widehat{x}}$ for any $\widehat{x}=(x, e) \in \widehat{M}$. Then $\left(\mathcal{H}_{x}\right)$ depends only on the isotopy class $\mathcal{I}_{x}$ of $x$.

Corollary 7.5 highlights one of the unique aspects of the study of Riemannian foliations, the duality between intrinsic and extrinsic geometry of leaves. That is, for $L_{y}$ near to $L_{x}$, we have that $L_{y} \sim L_{x}$ exactly when $L_{y}$ is diffeomorphic to $L_{x}$ via the orthogonal projection along leaves, and also that $L_{y} \sim L_{x}$ exactly when the holonomy group $\mathcal{H}_{\hat{y}}$ of $L_{y}$ is naturally conjugate to that of $L_{x}$.

\section{Foliations with Finite CATEGory}

Let $\mathcal{F}$ be a Riemannian foliation $\mathcal{F}$ of a compact manifold $M$. Then the $\mathbf{O}(q)$ equivariant LS category $\operatorname{cat}_{\mathbf{O}(q)}(\widehat{W})$ is finite, and by Corollaries 4.4 and 5.2

$$
\operatorname{cat}_{\Pi}^{e}(M, \mathcal{F})=\operatorname{cat}_{\mathbf{O}(q)}(\widehat{M}, \widehat{\mathcal{F}})=\operatorname{cat}_{\mathbf{O}(q)}(\widehat{W}) .
$$

In this section, we give geometric criteria for when $\operatorname{cat}_{巾}(M, \mathcal{F})$ is finite, and show that $\operatorname{cat}_{\phi}^{e}(M, \mathcal{F})=\operatorname{cat}_{\phi}(M, \mathcal{F})$ when these criteria are satisfied.

Theorem 1.4 follows from Propositions 8.1 and 8.4. Moreover, we obtain estimates for $\operatorname{cat}_{巾}(M, \mathcal{F})$ which extend the results of [17] for compact Hausdorff foliations.

Proposition 8.1. Let $Z_{x}$ be a locally minimal set for $\mathcal{F}$, and suppose that $H: U \times$ $[0,1] \rightarrow M$ is a transversely categorical open saturated set with $U \cap Z_{x} \neq \emptyset$. Then every leaf in $Z_{x}$ is compact.

Proof. Let $y \in U \cap Z_{x}$. By Proposition 7.3 every leaf of $\mathcal{F}$ in $Z_{x}$ is isotopic, so it suffices to show that there is some $z \in Z_{x}$ with $L_{z}$ compact.

Let $z=H_{1}(y)$, so the image of $H_{1}: U \rightarrow M$ is contained in the leaf $L_{z}$ of $\mathcal{F}$. As noted in section 2, the closure $\bar{L}_{y}$ is a compact minimal set for $\mathcal{F}$. By Lemma 2.2. we have that $\bar{L}_{y} \subset U$, hence by Theorem 1.3 of [37, the image $H_{1}\left(\bar{L}_{y}\right)$ is a compact leaf of $\mathcal{F}$; that is, $L_{z}$ is compact. It remains to show that $L_{z} \subset Z_{x}$. 
Lemma 8.2. Let $Z_{x}$ be a locally minimal set for $\mathcal{F}$ and $U$ an open saturated set such that $U \cap Z_{x} \neq \emptyset$. If $H: U \times[0,1] \rightarrow M$ is a foliated homotopy, then $H_{t}\left(Z_{x} \cap U\right) \subset Z_{x}$ for all $0 \leq t \leq 1$.

Proof. Let $\widehat{w} \in \widehat{W}$ be such that $Z_{x}=\pi\left(\widehat{\Upsilon}^{-1}\left(\mathcal{Z}_{\widehat{w}}\right)\right)$, and note that $\mathcal{Z}_{\widehat{w}}$ is a locally minimal set. Let $\widehat{U}=\pi^{-1}(U) \subset \widehat{M}$, and set $\mathcal{U}=\widehat{\Upsilon}(\widehat{U}) \subset \widehat{W}$.

Given $y \in U \cap Z_{x}$, choose a point $\widehat{\xi} \in \mathcal{U} \cap \mathcal{Z}_{\widehat{w}}$ so that $\widehat{\Upsilon}\left(\pi^{-1}(y)\right)=\widehat{\xi} \cdot \mathbf{O}(q)$.

By Proposition 4.1, the homotopy $H$ determines an $\mathbf{O}(q)$-equivariant homotopy $\mathcal{H}: \mathcal{U} \times[0,1] \rightarrow \widehat{W}$. By Proposition 6.15, $\widehat{\xi}_{t}=\mathcal{H}(\widehat{\xi}, t) \in \mathcal{Z}_{\widehat{w}}$ for all $0 \leq t \leq 1$, and thus $\pi\left(\widehat{\Upsilon}^{-1}\left(\widehat{\xi}_{t}\right)\right) \subset Z_{x}$. In particular, $L_{z}=\bar{L}_{z}=\pi\left(\widehat{\Upsilon}^{-1}\left(\widehat{\xi}_{1}\right)\right) \subset Z_{x}$.

The following is a direct consequence of Proposition 8.1, and generalizes the results of Colman in [15].

Corollary 8.3. Let $\mathcal{F}$ be a Riemannian foliation $\mathcal{F}$ of a compact manifold $M$ with $\operatorname{cat}_{\phi}(M, \mathcal{F})$ finite. Then every local minimal set $Z_{i}$ for $\mathcal{F}$ is a union of compact leaves.

The following converse to Corollary 8.3 gives our criterium for $\operatorname{cat}_{巾}(M, \mathcal{F})<\infty$.

Proposition 8.4. Let $\mathcal{F}$ be a Riemannian foliation $\mathcal{F}$ of a compact manifold $M$ such that every closed isotopy stratum $I_{x}$ for $\mathcal{F}$ consists of compact leaves. Then $\operatorname{cat}_{\pitchfork}(M, \mathcal{F})=\operatorname{cat}_{\pitchfork}^{e}(M, \mathcal{F})$ and hence is finite.

Proof. Let $\left\{\mathcal{H}_{\ell}: \mathcal{U}_{\ell} \times[0,1] \rightarrow \widehat{W} \mid 1 \leq \ell \leq k\right\}$ be an $\mathbf{O}(q)$-categorical covering of $\widehat{W}$, for $k=\operatorname{cat}_{\mathbf{O}(q)}(\widehat{W})$. Let $\left\{\widehat{w}_{1}, \ldots, \widehat{w}_{k}\right\} \subset \widehat{W}$ be such that $\mathcal{H}_{\ell}\left(\mathcal{U}_{\ell}\right) \subset \widehat{w}_{\ell} \mathbf{O}(q)$. For each $1 \leq \ell \leq k$, let $\widehat{x}_{\ell} \in \widehat{\Upsilon}^{-1}\left(\widehat{w}_{\ell}\right)$ and set $x_{\ell}=\pi\left(\widehat{x}_{\ell}\right)$ and $L_{\ell}=L_{x_{\ell}} \subset M$.

By Proposition 6.16 we can assume that $\mathcal{Z}_{\widehat{w}_{\ell}}$ is locally minimal for each $1 \leq$ $\ell \leq k$. By Corolllary 7.4 the corresponding isotopy stratum $I_{x_{\ell}} \subset M$ for $\mathcal{F}$ is closed. By assumption, all leaves in $I_{x_{\ell}}$ are compact. By choice, $L_{x_{\ell}} \subset Z_{x_{\ell}}$, hence is compact.

Let $U_{\ell}=\pi\left(\widehat{\Upsilon}^{-1}\left(\mathcal{U}_{\ell}\right)\right)$ and $H_{\ell}: U_{\ell} \times[0,1] \rightarrow M$ be the $\mathcal{F}$-foliated homotopy corresponding to $\mathcal{H}_{\ell}$. Then $H_{1}: U_{\ell} \rightarrow \bar{L}_{\ell}=L_{\ell}$ is a compact leaf.

The results and techniques of the previous sections and those above yield a strong estimate for the transverse LS category of Riemannian foliations. Proposition 7.3 identifies the isotropy stratification of $\mathcal{F}$ with the $\mathcal{Z}$-stratification of the $\mathbf{O}(q)$-action on $\widehat{W}$. Then $\left\{I_{0}, I_{1}, \ldots, I_{K}\right\}$ is an enumeration of the isotropy strata for $\mathcal{F}$, so that $I_{\ell}$ is a closed submanifold exactly when $1 \leq \ell \leq \alpha=\alpha(M, \mathcal{F})$. Then by Lemma 8.2, Theorem 6.17 and equation (41) we obtain

Theorem 8.5. Let $\mathcal{F}$ be a Riemannian foliation of a compact manifold $M$. Then

$$
\alpha(M, \mathcal{F}) \leq \sum_{\ell=1}^{\alpha_{\mathcal{F}}(M)} \operatorname{cat}_{巾}^{e}\left(I_{\ell}, \mathcal{F} \mid I_{\ell}\right) \leq \operatorname{cat}_{\phi}^{e}(M, \mathcal{F}) \leq \sum_{\ell=0}^{K} \operatorname{cat}_{巾}^{e}\left(I_{\ell}, \mathcal{F} \mid I_{\ell}\right) .
$$

Moreover, if each $I_{\ell}$ for $1 \leq \ell \leq \alpha_{\mathcal{F}}(M)$ is a union of compact leaves, then

$$
\operatorname{cat}_{\pitchfork}(M, \mathcal{F})=\operatorname{cat}_{巾}^{e}(M, \mathcal{F}) ;
$$

hence the estimates (49) also hold for the transverse LS category.

Theorem 8.5 is a complete generalization of the estimate given by Theorem 6.1 in [17] for the tranverse LS category of compact Hausdorff foliations. 


\section{Critical points}

A principal application of the LS-category invariant for a compact manifold $N$ is to give a lower bound estimate on the number of critical points for a $C^{1}$-function $f: N \rightarrow \mathbb{R}$ (see [19, 25, 26, 43, 50, 59, 60]). When there is a compact Lie group $G$ acting on $N$ and the function $f$ is invariant for the $G$-action, then one seeks to count the number of critical $G$-orbits for $f$. The simplest approach would be to consider the induced map on the quotient space, $\bar{f}: N / G \rightarrow \mathbb{R}$, and to then estimate the number of critical points of $\bar{f}$. There are two problems with this approach, the first being that the quotient space $N / G$ need not be a manifold, so the classical Lusternik-Schnirelmann theory does not apply. The second, more subtle difficulty, is seen in various examples where the category of $N / G$ is "too small". In other words, the LS category of the quotient space $N / G$ is not the best estimate for the number of critical $G$-orbits of $f$.

The well-known solution to these difficulties is to consider the set of critical points for $f$ as a $G$-space, and then the $G$-category $\operatorname{cat}_{G}(N)$ provides a lower bound for the number of critical $G$-orbits 4, 25, 26, 151. Moreover, we always have that $\operatorname{cat}_{G}(N) \geq \operatorname{cat}(N / G)$, and examples show that $\operatorname{cat}_{G}(N)$ generically provides the optimal lower bound estimate for the number of critical $G$-orbits.

Now consider a $C^{1}$-function $f: M \rightarrow \mathbb{R}$ on a closed manifold $M$ with Riemannian foliation $\mathcal{F}$. Recall that $f$ is $\mathcal{F}$-basic if it is constant along leaves of $\mathcal{F}$. Then the differential $d f: T M \rightarrow \mathbb{R}$ is a basic 1-form, so it makes sense to speak of the "critical leaves" for $\mathcal{F}$, those for which the gradient $\nabla f=0$. In her thesis, Colman proved that the Lusternik-Schnirelmann estimate generalizes to the case where all leaves of $\mathcal{F}$ are compact (see section 5 of [18]). That is, Colman showed that the transverse category $\operatorname{cat}_{\pitchfork}(M, \mathcal{F})$ is a lower bound for the number of critical leaves. However, for Riemannian foliations with non-compact leaves, both the techniques of [18] and even the definition of $\operatorname{cat}_{巾}(M, \mathcal{F})$ run into difficulties.

Let $\mathcal{F}$ be an arbitrary Riemannian foliation of the closed manifold $M$. Let $f$ be a $C^{1}$-function which is $\mathcal{F}$-basic; hence $f$ is also constant on the leaves of the singular Riemannian foliation $\mathcal{E}$ of $M$ defined by the closures of the leaves of $\mathcal{F}$. The differential $d f: T M \rightarrow \mathbb{R}$ is a basic 1-form, so if $L$ is a critical, then the leaf $\bar{L}$ of $\mathcal{E}$ is a union of critical leaves of $\mathcal{F}$.

The techniques of this paper allow us to prove a Lusternik-Schnirelmann estimate for the number of critical leaf closures of $f$. First, define the lift $\widehat{f}=f \circ \pi: \widehat{M} \rightarrow \mathbb{R}$, which is a basic $C^{1}$-function for $\widehat{\mathcal{F}}$.

Note that a leaf $\widehat{L}_{\widehat{x}}$ of $\widehat{\mathcal{F}}$ will be critical for $\widehat{f}$ if and only if $L_{x}$ is critical for $f$, and the closure $\bar{L}_{\widehat{x}}$ of a critical leaf is a critical submanifold for $d \widehat{f}$. Moreover, the function $\widehat{f}$ is $\mathbf{O}(q)$-invariant, so we can estimate the number of critical leaf closures for $f$ in terms of the $\mathbf{O}(q)$-invariant critical submanifolds of $\widehat{f}$ in $\widehat{M}$.

The $C^{1}$-function $\widehat{f}$ descends to an $\mathbf{O}(q)$-invariant $C^{1}$-function $\widehat{\phi}: \widehat{W} \rightarrow \mathbb{R}$, where the critical orbits for $\widehat{\phi}$ correspond exactly to the critical leaf closures of $\widehat{f}$, and hence to the closures of the critical leaves for $f$.

Apply the results of equivariant LS-category theory for the action of $\mathbf{O}(q)$ on the closed manifold $\widehat{W}$ to estimate the number of critical $\mathbf{O}(q)$-orbits for $\widehat{\phi}$.

Theorem 9.1 (Fadell [25], Marzantowicz [51]). Let $G$ be a compact Lie group, and $R: N \times G \rightarrow N$ a smooth right action on a closed manifold $N$. If $\widehat{\phi}: N \rightarrow \mathbb{R}$ is a 
$C^{1}$-function which is $G$-invariant, then $\operatorname{cat}_{G}(N)$ is a lower bound on the number of critical orbits for $\widehat{\phi}$.

By Theorem 1.6 and the above discussion, we obtain the claim of Theorem 1.1;

Theorem 9.2. Let $f: M \rightarrow \mathbb{R}$ be a $C^{1}$-map which is constant along the leaves of $\mathcal{F}$. Then the number of critical leaf closures for $f$ is bounded below by cate $(M, \mathcal{F})$.

The great advantage of this indirect approach to the study of critical point theory for $f$ is that the technical estimates required can be done in the context of a compact group action. In the compact case, the results are already well-known, while in the case of a foliation with non-compact leaves, the new techniques required essentially duplicate some of the key geometric aspects from this paper.

\section{EXAMPLES}

In theory, the Molino Structure Theory gives a complete procedure for constructing Riemannian foliations on a given compact manifold. In practice, however, it has proven effective only for foliations of low codimension, usually three or less [71, 72, 56, or with other restrictions on their topology [28].

In this last section, we discuss three general methods for constructing a Riemannian foliation on a compact manifold: isometric Lie group actions; the group suspension construction applied to an isometric action of a finitely generated group; and the various blow-up constructions for singular Riemannian foliations [1, 2, 56, 57. In each case, the examples will be used to illustrate results of this paper.

Note that for open manifolds, there is an important fourth method, which realizes a Riemannian pseudogroup as a Riemannian foliation of an open manifold [29, 36]. These cases are not discussed, as very little is known of their transverse LS-category.

Example 10.1. Compact Hausdorff foliations and finite group actions.

A foliation $\mathcal{F}$ is said to be compact Hausdorff if all of its leaves are compact, and the leaf space $M / \mathcal{F}$ is Hausdorff with the quotient topology. Epstein [23] and Millett [53] proved that a compact Hausdorff foliation $\mathcal{F}$ admits a projectable Riemannian metric, and hence is Riemannian.

For each $x \in M$, the holonomy $\mathcal{H}_{x}$ of the leaf $L_{x}$ is always a finite group; hence all leaves of the lifted foliation $\widehat{\mathcal{F}}$ of the orthonormal frame bundle $\widehat{M}$ are also compact. The lifted foliation $\widehat{\mathcal{F}}$ is thus defined by the fibration $\widehat{\Upsilon}: \widehat{M} \rightarrow \widehat{W}$.

The quotient space $W=M / \mathcal{F}$ is a Satake manifold [64], or generalized orbifold, as every point is modeled either on $\mathbb{R}^{q}$ or by a quotient of $\mathbb{R}^{q}$ by a finite isometry group given by the holonomy of the leaf fiber. The quotient map $\widehat{\pi}: \widehat{W} \rightarrow W$ is an "O$(q)$-desingularization" of $W$. The regular $\mathbf{O}(q)$-orbits correspond to the leaves of $\mathcal{F}$ without holonomy. A leaf $L_{x}$ with non-trivial holonomy for $\mathcal{F}$ corresponds to an orbit $\widehat{w} \cdot \mathbf{O}(q)$ on $\widehat{W}$ with a finite non-trivial isotropy group $\mathbf{H}_{\widehat{w}}$. Hence, every orbit of $\mathbf{O}(q)$ is either regular or exceptional, but there are no singular orbits.

The exceptional set $E_{\mathcal{F}}$ of a compact foliation $\mathcal{F}$, introduced by Epstein and Millett, is the union of all leaves with holonomy. The Epstein filtration of $E_{\mathcal{F}}$ is inductively defined using the holonomy groups of its leaves. Moreover, the celebrated "Moving Leaf Lemma" of Edwards, Millett, and Sullivan 22] characterizes the strata of the Epstein filtration in terms of isotopy of the compact leaves of $\mathcal{F}$. Thus, the stratification of $M$ derived from the Epstein filtration corresponds exactly to the isotropy stratification of $M$ defined in section 7 
One of the motivations for this current work was to find a new approach to the results for compact Hausdorff foliations in [17, an approach which generalizes to all Riemannian foliations. For example, Theorem 5.3 of [17] proves the Epstein filtration is invariant under $\mathcal{F}$-foliated homotopy. This result is now a direct consequence of Proposition 7.3 and the results of section 6 of this paper. Moreover, Theorem 8.5 above implies the estimate of Theorem 6.1 in [17.

Example 10.1.1. Colman has given numerous examples of compact Hausdorff foliations along with the calculations of their tranverse LS category (see the papers [14, 16, 17]). The standard method of construction is to start with a fibration $\widetilde{\pi}: \widetilde{M} \rightarrow \widetilde{W}$ of compact manifolds, and assume that $\widetilde{\pi}$ is equivariant with respect to a finite group $\Gamma$ which acts freely on $\widetilde{M}$. Then the foliation of $\widetilde{M}$ by the fibers of $\widetilde{\pi}$ descends to a compact Hausdorff foliation of $M$, whose leaf space $W=\widetilde{W} / \Gamma$ is thus a good orbifold. Colman has also given examples of compact Hausdorff foliations whose leaf space $M / \mathcal{F}$ is a bad orbifold.

Example 10.2. Isometric flows.

Isometric flows are perhaps the most well-known examples of Riemannian foliations (after fibrations). Let $(M, g)$ be a compact, connected Riemannian manifold, and $\phi: M \times \mathbb{R}^{p} \rightarrow M$ a non-singular isometric action of $\mathbb{R}^{p}$. (The case $p=1$ corresponds to an isometric flow on $M$.) The orbits of $\phi$ define a Riemannian foliation $\mathcal{F}_{\phi}$ for which the metric $g$ is projectable. The geometric and topological properties of this class of Riemannian foliations had been studied by many authors [10, 24, 28, 33, 34.

The leaves of the lifted foliation $\widehat{\mathcal{F}}$ are given by a free $\mathbb{R}^{p}$ isometric action on $\widehat{M}$. The closure of the image of $\mathbb{R}^{p}$ in $\operatorname{Isom}(\widehat{M})$ is a torus $\mathbb{T}^{k}$ for some $k>p$, thus the foliation $\widehat{\mathcal{E}}$ is defined by a free isometric action of $\mathbb{T}^{k}$ on $\widehat{M}$. This class of examples reveals many of the properties of the transverse category theory for Riemannian foliations, and we give several examples to illustrate various phenomena.

Example 10.2.1. The canonical example of an "irrational flow on the torus" is formulated generally as follows. Let $M=\mathbb{T}^{n}$ be the $n$-torus, considered as the quotient $\mathbb{T}^{n}=\mathbb{R}^{n} / \mathbb{Z}^{n}$ by the integer lattice. Choose $1 \leq p<n$ and a real matrix $A^{p \times n}$ such that $A A^{T}$ is invertible. Then $A$ defines an injective map $\mathbf{A}: \mathbb{R}^{p} \rightarrow \mathbb{R}^{n}$, and thus yields an isometric affine action $\phi_{A}: \mathbb{R}^{p} \times \mathbb{T}^{n} \rightarrow \mathbb{T}^{n}$. The orbits of $\phi_{A}$ are the affine planar leaves of $\mathcal{F}_{A}$. All leaves of $\mathcal{F}_{A}$ are regular, as there is no holonomy.

If all entries of $A$ are rational numbers, then the leaves of $\mathcal{F}_{A}$ are compact tori; otherwise, the leaves have closures which are embedded tori $\xi_{A}: \mathbb{T}^{k} \subset \mathbb{T}^{n}$ for some $p<k \leq n$. Thus, $\operatorname{cat}_{巾}\left(\mathbb{T}^{n}, \mathcal{F}_{A}\right)=\infty$ unless $A$ is a rational matrix. On the other hand, the essential transverse category is equal to the category of the foliation $\mathcal{E}_{A}$ obtained from the closures of the leaves. The leaf space $\mathbb{T}^{n} / \mathcal{E}_{A} \cong \mathbb{T}^{n} / \xi_{A}\left(\mathbb{T}^{k}\right)$ is a torus of dimension $n-k$, hence cat $\cos ^{e}\left(\mathbb{T}^{n}, \mathcal{F}_{A}\right)=\operatorname{cat}\left(\mathbb{T}^{n-k}\right)=n-k+1$.

Example 10.2.2. The previous examples of isometric flows can be embedded into compact space forms. We begin with the simplest examples of this.

Let $\vec{\alpha}=\left(\alpha_{0}, \alpha_{1}, \ldots, \alpha_{n}\right) \in \mathbb{R}^{n+1}$ and let $M$ be the unit $2 n+1$ sphere

$$
M=\mathbb{S}^{2 n+1}=\left\{x=\left.\left[z_{0}, z_{1}, \ldots, z_{n}\right]|| z_{0}\right|^{2}+\cdots+\left|z_{n}\right|^{2}=1\right\} .
$$


Define an isometric $\mathbb{R}$-action on $\mathbb{S}^{2 n+1}$ by

$$
\phi_{t}\left(\left[z_{0}, \ldots, z_{n}\right]\right)=\left[e^{2 \pi \alpha_{0} t \sqrt{-1}} z_{0}, \ldots, e^{2 \pi \alpha_{n} t \sqrt{-1}} z_{n}\right] .
$$

The orbits of $\phi_{t}$ define the leaves of the foliation $\mathcal{F}_{\vec{\alpha}}$. With the assumption that the numbers $\left\{1, \alpha_{0}, \cdots, \alpha_{n}\right\}$ are linearly independent over $\mathbb{Q}$, then the leaves of $\overline{\mathcal{F}_{\vec{\alpha}}}$ are defined by the action of the compact abelian group $\mathbb{T}^{n+1}=\mathbb{S}^{1} \times \cdots \mathbb{S}^{1}$ acting diagonally.

There are precisely $n+1$ locally minimal $\mathcal{F}_{\vec{\alpha}}$-isotopy classes, corresponding to the orbits of the points $\vec{e}_{i}=[0, \ldots, 1, \ldots, 0]$, which are isolated circles. Thus, $\operatorname{cat}_{\phi}\left(\mathbb{S}^{2 n+1}, \mathcal{F}_{\vec{\alpha}}\right) \geq n+1$. It is also easily seen that for each point $\vec{e}_{i}$ there is a flow-equivariant retraction of the open set $\mathcal{U}_{i}=\left\{\left[z_{0}, \ldots, z_{n}\right] \in \mathbb{S}^{2 n+1} \mid z_{i} \neq 0\right\}$ to the orbit of $\vec{e}_{i}$. Hence cat ${ }_{巾}\left(\mathbb{S}^{2 n+1}, \mathcal{F}_{\vec{\alpha}}\right)=n+1$.

It is possible to construct a wide variety of variations on this example, based on the general setup where $G$ is a connected, compact Lie group and $K \subset G$ is a closed subgroup, and we set $M=G / K$. Let $n$ denote the $\mathbb{R}$-rank of $G$, so there is a locally free action $\Phi_{t}: \mathbb{R}^{n} \times G \rightarrow G$ whose orbit through the identity $e \in G$ is a maximal torus $\mathbb{T}^{n} \subset G$.

Choose $1 \leq p \leq n$ and a real matrix $A^{p \times n}$ such that $A A^{T}$ is invertible. Then $A$ defines an injective map $\mathbf{A}: \mathbb{R}^{p} \rightarrow \mathbb{R}^{n}$, and thus yields an isometric affine action $\phi_{A}: \mathbb{R}^{p} \times \mathbb{T}^{n} \rightarrow \mathbb{T}^{n}$

For $\vec{v} \in \mathbb{R}^{p}$ and $x \in G / K$, define the action $\phi_{A}(x, \vec{v})=\Phi_{t}\left(x, \phi_{A}(\vec{v})\right)$ so that we obtain a $p$-dimensional foliation $\mathcal{F}_{A}$ on the homogenous space $G / K$.

The closure of the orbit of $\phi_{A}$ through the identity is a compact $k$-torus $\mathbb{T}_{A}^{k} \subset \mathbb{T}^{n}$ for some $p<k \leq n$. The foliation $\mathcal{E}_{A}-$ defined by the closures of the leaves of $\mathcal{F}_{A}$ - is given by the orbits of the closed subgroup $\mathbb{T}_{A}^{k}$ on $G / K$.

Note that in this generality there is no assurance that $\mathcal{F}_{A}$ has any compact leaves, hence generically one has cat ${ }_{\pitchfork}\left(G / K, \mathcal{F}_{A}\right)=\infty$. On the other hand, $\operatorname{cat}_{\pitchfork}^{e}\left(G / K, \mathcal{F}_{A}\right)$ equals the equivariant category of $G / K$ for the left action of the compact Lie subgroup $\mathbb{T}_{A}^{k} \subset G$. The calculation of cat ${ }_{巾}^{e}\left(G / K, \mathcal{F}_{A}\right)$ then follows by methods of the theory of compact Lie groups, and has applications to the residue theory for the secondary classes of $\mathcal{F}_{A}$ [38, 48, 49, 71, 72].

Example 10.3. Products.

If $\left(M_{1}, \mathcal{F}_{1}\right)$ and $\left(M_{2}, \mathcal{F}_{2}\right)$ are Riemannian foliations of compact manifolds with leaf dimensions $p_{1}$ and $p_{2}$, respectively, then the product manifold $M=M_{1} \times M_{2}$ has a Riemannian foliation $\mathcal{F}=\mathcal{F}_{1} \times \mathcal{F}_{2}$ whose leaves have dimension $p=p_{1}+p_{2}$.

For example, suppose that $M_{2}=\mathbb{T}^{n_{2}}$ has a linear foliation as in Example 10.21 with all leaves dense. Then for any Riemannian foliation $\left(M_{1}, \mathcal{F}_{1}\right)$ the product foliation $\mathcal{F}$ on $M_{1} \times \mathbb{T}^{n_{2}}$ has no compact leaves, hence cat $\cot (M, \mathcal{F})=\infty$. On the other hand, $\operatorname{cat}_{币}^{e}(M, \mathcal{F})=\operatorname{cat}_{币}^{e}\left(M_{1}, \mathcal{F}_{1}\right)$.

Example 10.4. Suspension and compact Lie group actions.

Given an action of a finitely generated group on a compact $q$-dimensional manifold, $\alpha: \Gamma \times N \rightarrow N$, the suspension construction yields a foliation $\mathcal{F}_{\alpha}$ of codimension $q$ whose transverse holonomy group is globally defined by the action (for example, see [8, 9]). When the given action is isometric, this yields a Riemannian foliation, and provides a large class of examples. We use this construction to realize 
the orbit structure of every compact Lie group action as the transverse geometry of some Riemannian foliation.

Example 10.4.1. Let $\left\{\gamma_{1}, \ldots, \gamma_{d}\right\}$ be a set of generators for $\Gamma$. A left isometric action of $\Gamma$ on $N$ is equivalent to a representation $\alpha: \Gamma \rightarrow \operatorname{Isom}(N)$, where each $\alpha\left(\gamma_{i}\right)$ acts isometrically on the left on $N$.

Let $B$ be a compact connected Riemannian manifold with basepoint $b_{0} \in B$ such that the fundamental group $\Pi=\pi_{1}\left(B, b_{0}\right)$ admits a surjection $\beta: \Pi \rightarrow \Gamma$. For example, one can let $B=\Sigma_{d}$ be a closed Riemann surface with genus $d$. There is a surjection $\Pi \rightarrow \mathbb{F}^{d}=\mathbb{Z} * \cdots * \mathbb{Z}$ onto the non-abelian free group on $d$ generators. The choice of the generators for $\Gamma$ defines a surjection $\mathbb{F}^{d} \rightarrow \Gamma$, and the composition $\beta: \Pi \rightarrow \Gamma$ is then a surjection.

The universal cover $\widetilde{B} \rightarrow B$ has a right isometric action by $\Pi$, acting via deck transformations.

Consider the product manifold $\widetilde{B} \times N$ with the product foliation $\widetilde{\mathcal{F}}$ whose leaves are the "horizontal slices" $\widetilde{B} \times\{x\}$ for $x \in N$. The Riemannian metrics on $\widetilde{B}$ and $N$ define the product metric on $\widetilde{B} \times N$.

Define an action of $\Pi$ on $\widetilde{B} \times N$ by specifying, for $\lambda \in \Pi,(b, x) \cdot \lambda=(b \cdot \lambda, \alpha$ 。 $\left.\beta\left(\lambda^{-1}\right) \cdot x\right)$.

Both the product foliation and the product Riemannian metric on $\widetilde{B} \times N$ are invariant under this action, so the product foliation descends to a Riemannian foliation $\mathcal{F}_{\alpha}$ of $M=\widetilde{B} \times_{\Pi} N$. Note that there is an embedding $\iota_{0}: N \rightarrow M$ given by $\iota(x)=\left[b_{0}, x\right]$ where $[b, x]$ represents the equivalence class of the pair $(b, x)$ in $M$. Let $N_{0} \subset M$ denote the image of this map.

The group of isometries $\operatorname{Isom}(N)$ is compact, so the closure $G=\overline{\alpha(\Gamma)} \subset$ $\operatorname{Isom}(N)$ is a compact Lie subgroup. Let $\mathcal{E}_{\alpha}$ denote the singular Riemannian foliation of $M$ by the closures of the leaves of $\mathcal{F}_{\alpha}$. Then the leaves of $\mathcal{E}_{\alpha} \cap N_{0}$ are precisely given by the orbits of $G$.

Example 10.4.2. Let $G$ be a compact connected Lie group, and $\varphi: G \times N \rightarrow N$ a smooth isometric action on a compact Riemannian manifold $N$ of dimension $q$. The orbits of $\varphi$ define a singular Riemannian foliation $\mathcal{E}_{\varphi}$ of $N$ [33, 34, 56, 57. Ken Richardson [62] showed that there always exists a Riemannian foliation $\mathcal{F}_{\varphi}$ of a compact manifold $M$ such that the singular Riemannian foliation defined by the closures of the leaves of $\mathcal{F}_{\varphi}$ is transversally equivalent to $\mathcal{E}_{\varphi}$. We recall this argument.

Let $\Gamma \subset G$ be a finitely generated dense subgroup; such a subgroup always exists by a clever argument of Richardson 62. The restriction of $\varphi$ to the subgroup defines a representation $\alpha: \Gamma \rightarrow \operatorname{Isom}(N)$.

Use the suspension construction as in Example 10.42 to obtain a Riemannian foliation $\left(M, \mathcal{F}_{\alpha}\right)$ such that the image $\alpha(\Gamma) \subset \operatorname{Isom}(N)$ has closure precisely the compact Lie subgroup $G$. The orbit type stratification of the $G$-action on $N$ equals the stratification of the transversal $N_{0}$ induced by the closures of the leaves of $\mathcal{F}_{\varphi}$.

There is a particular case of this construction which yields some very interesting examples. Let $G=\mathbf{S U}(n)$ be the group of $n \times n$ special unitary matrices. Let $N=\mathbf{S U}(n)$ be the group itself, and let the action $\varphi$ be the adjoint, so that $\varphi(A): \mathbf{S U}(n) \rightarrow \mathbf{S U}(n)$ is given by $\varphi(A)(B)=A^{-1} B A$. Let $\mathcal{F}_{\alpha}$ denote the resulting suspension foliation. Then $\operatorname{cat}_{\pitchfork}^{e}\left(M, \mathcal{F}_{\alpha}\right)=n$, based on the calculations of [38]. Note that the codimension of $\mathcal{F}_{\alpha}$ is the dimension of $\mathbf{S U}(n)$ so that $q=n^{2}-1$. 
The suspension of the adjoint action of $\mathbf{S U}(n)$ on itself yields a foliation with no exceptional orbits. This is not the case with the groups $\mathbf{S O}(n)$. In fact, for this case, there are isolated exceptional orbits, so by Colman's results, the transverse category of the suspended foliation will be infinite. However, the essential transverse category will be finite, and its calculation is a very important problem, as it yields estimates for the category of the groups $\mathbf{S O}(n)$ themselves [38].

\section{REFERENCES}

[1] M.M. Alexandrino, Singular Riemannian foliations with sections, Illinois J. Math., 48:1163-1182, 2004. MR2113670 (2005i:53025)

[2] M.M. Alexandrino and D. Töben, Singular Riemannian foliations on simply connected spaces, Differential Geom. Appl., to appear. MR2231053 (2007d:53037)

[3] R. Ayala, F.F. Lasheras and A. Quintero, The equivariant category of proper G-spaces, Rocky Mountain J. Math., 31:1111-1132, 2001. MR.1895288(2003b:55002)

[4] T. Bartsch, Topological methods for variational problems with symmetries, Lect. Notes in Math. Vol. 1560, Springer-Verlag, Berlin, 1993. MR.1295238 (96a:58078)

[5] R. Blumenthal and J. Hebda, Ehresmann connections for foliations, Indiana Univ. Math. J., 33:597-611, 1984. MR749317 (85m:53024)

[6] R. Blumenthal and J. Hebda, Complementary distributions which preserve the leaf geometry and applications to totally geodesic foliations, Quart. J. Math. Oxford Ser. (2), 35:383392, 1984. MR767769 (86e:53021)

[7] G. Bredon, Introduction to compact transformation groups, Pure and Applied Mathematics, Vol. 46, Academic Press, New York, 1972. MR0413144 (54:1265)

[8] C. Camacho and A. Lins Neto, Geometric Theory of Foliations, translated from the Portuguese by Sue E. Goodman, Progress in Mathematics, Birkhäuser Boston, Inc., Boston, MA, 1985. MR824240 (87a:57029)

[9] A. Candel and L. Conlon, Foliations I, Amer. Math. Soc., Providence, RI, 2000.

[10] Y. Carrière, Flots riemanniens, in Transversal structure of foliations (Toulouse, 1982), Asterisque, 116, Société Mathématique de France, 1984, 31-52. MR.755161 (86m:58125a)

[11] K.-T. Chen, Iterated path integrals, Bull. Amer. Math. Soc., 83:831-879, 1977. MR0454968 (56:13210)

[12] H. Colman, Categoría LS en foliaciones, Publicaciones del Departamento de Topología y Geometría, no. 93, Universidade de Santiago de Compostele, 1998.

[13] H. Colman, LS-categories for foliated manifolds, in Foliations: Geometry and Dynamics (Warsaw, 2000), World Scientific Publishing Co. Inc., River Edge, N.J., 2002:17-28. MR:1882763 (2002m:55007)

[14] H. Colman, Equivariant LS-category for finite group actions, in Lusternik-Schnirelmann category and related topics (South Hadley, MA, 2001), Contemp. Math. Vol. 316, Amer. Math. Soc., Providence, R.I., 2002, 35-40. MR.1962151 (2004c:55002)

[15] H. Colman, Transverse Lusternik-Schnirelmann category of Riemannian foliations, Topology Appl., 141:187-196, 2004. MR2058687(2005e:55004)

[16] H. Colman, Lusternik-Schnirelmann category of Orbifolds, preprint, 2006.

[17] H. Colman and S. Hurder, LS-category of compact Hausdorff foliations, Trans. Amer. Math. Soc., 356:1463-1487, 2004. MR2034314 (2004i:55004)

[18] H. Colman and E. Macias, Transverse Lusternik-Schnirelmann category of foliated manifolds, Topology Vol. 40 (2) (2001), 419-430. MR1808226 (2002c:55010)

[19] O. Cornea, G. Lupton, J. Oprea, and D. Tanré, Lusternik-Schnirelmann category, Mathematical Surveys and Monographs 103, American Mathematical Society, 2003. MR 1990857 (2004e:55001)

[20] M.W. Davis, Smooth G-manifolds as collections of fiber bundles, Pacific J. Math., 77:315363, 1978. MR510928 (80b:57034)

[21] J.J. Duistermaat and J.A.C. Kolk, Lie Groups, Universitext, Springer-Verlag, Berlin, 2000. MR1738431 (2001j:22008) 
[22] R. Edwards, K. Millett, and D. Sullivan, Foliations with all leaves compact, Topology, 16:13-32, 1977. MR0438353 (55:11268)

[23] D.B.A. Epstein, Foliations with all leaves compact, Ann. Inst. Fourier (Grenoble), 26:265-282, 1976. MR0420652 (54:8664)

[24] D. B. A. Epstein, Transversely hyperbolic 1-dimensional foliations, in Transversal structure of foliations (Toulouse, 1982), Asterisque, 116, Société Mathématique de France, 1984, 53-69. MR755162 (86m:58125b)

[25] E. Fadell, The equivariant Lusternik-Schnirelmann method for invariant functionals and relative cohomological index theories, in Topological methods in nonlinear analysis, Sém. Math. Sup. Vol. 95, ed. A. Granas, Presses Univ. Montréal, Montreal, QC, 1985. MR801933 (87b:58018)

[26] E. Fadell and S. Husseini, Relative cohomological index theories, Adv. Math., 64:1-31, 1987. MR879854 (88f:55006)

[27] Y. Felix, S. Halperin, and J.-C. Thomas, Rational homotopy theory, Graduate Texts in Mathematics, Vol. 205, Springer-Verlag, New York and Berlin, 2001. MR 1802847 (2002d:55014)

[28] É. Ghys, Feuilletages riemanniens sur les variétés simplement connexes, Ann. Inst. Fourier (Grenoble), 34:203-223, 1984. MR766280 (86c:57025)

[29] A. Haefliger, Homotopy and integrability, in Manifolds-Amsterdam 1970 (Proc. Nuffic Summer School), Lecture Notes in Mathematics, Vol. 197, Springer, Berlin, 1971, pages 133-163. MR0285027 (44:2251)

[30] A. Haefliger, Pseudogroups of local isometries, in Differential geometry (Santiago de Compostela, 1984), Res. Notes in Math., Vol. 131:174-197, Pitman, Boston, MA, 1985. MR864868 (88i:58174)

[31] A. Haefliger, Leaf closures in Riemannian foliations, in A Fête of Topology, Academic Press, Boston, MA, 1988, 3-32. MR928394 (89c:57033)

[32] A. Haefliger, Feuilletages riemanniens, in Séminaire Bourbaki, Vol. 1988/89, Asterisque, 177-178, Société Mathématique de France, 1989, 183-197. MR1040573 (91e:57047)

[33] A. Haefliger and É. Salem, Riemannian foliations on simply connected manifolds and actions of tori on orbifolds, Illinois J. Math., 34:706-730, 1990. MR.1062771 (92c:57025)

[34] A. Haefliger and É. Salem, Actions of tori on orbifolds, Ann. Global Anal. Geom., 9:3759, 1991. MR 1116630 (92f:57047)

[35] W.-C. Hsiang and W.-Y. Hsiang, Differentiable actions of compact connected classical groups. I, Amer. J. Math., 89:705-786, 1967. MR0217213 (36:304)

[36] S. Hurder, On the homotopy and cohomology of the classifying space of Riemannian foliations, Proc. Amer. Math. Soc., 81:485-489, 1981. MR.597668 (82j:57029)

[37] S. Hurder, Category and compact leaves, Topology Appl., 153:2135-2154, 2006. MR2239077 (2008a:57027)

[38] S. Hurder and D. Töben, The equivariant LS-category of polar actions, Topology Appl., to appear. MR2239077(2008a:57027)

[39] S. Hurder and D. Töben, Residues and transverse LS category for Riemannian foliations, in preparation, 2007.

[40] S. Hurder and P. Walczak, Compact foliations with finite transverse LS category, Jour. Math. Soc. Japan, to appear.

[41] P. Iglésias, Connexions et difféologie, in Aspects dynamiques et topologiques des groupes infinis de transformation de la mécanique (Lyon, 1986), Travaux en Cours Vol. 25, Hermann, Paris, 1987, 61-78. MR906897 (88k:58019)

[42] I.M. James, On category, in the sense of Lusternik-Schnirelmann, Topology 17:331-348, 1978. MR516214 (80i:55001)

[43] I.M. James, Lusternik-Schnirelmann Category, Chapter 27, Handbook of Algebraic Topology, 1995, 1293-1310. MR1361912 (97a:55003)

[44] K. Jänich, On the classification of $O(n)$-manifolds, Math. Ann., 176:53-76, 1968. MR0226674 (37:2261)

[45] K. Kawakubo, The theory of transformation groups, translated from the 1987 Japanese edition, Oxford University Press, New York, 1991. MR.1150492 (93g:57044)

[46] W. Krawcewicz and W. Marzantowicz, Lusternik-Schnirelman method for functionals invariant with respect to a finite group action, J. Differential Equations, 85:105-124, 1989. MR1052330 (91g:58048) 
[47] R. Langevin and P. Walczak, Transverse Lusternik-Schnirelmann category and non-proper leaves, in Foliations: Geometry and Dynamics (Warsaw, 2000), World Scientific Publishing Co. Inc., River Edge, N.J., 2002:351-354. MR1882778 (2003d:57051)

[48] C. Lazarov and J. Paternack, Secondary characteristic classes for Riemannian foliations, J. Differential Geometry, 11:365-385, 1976. MR0445513 (56:3853)

[49] C. Lazarov and J. Paternack, Residues and characteristic classes for Riemannian foliations, J. Differential Geometry, 11:599-612, 1976. MR0445514 (56:3854)

[50] L. Lusternik and L. Schnirelmann, Méthodes topologiques dans les Problèmes Variationnels. Hermann, Paris, 1934.

[51] W. Marzantowicz, A G-Lusternik-Schnirelman category of space with an action of a compact Lie group, Topology, 28:403-412, 1989. MR1030984 (91c:55002)

[52] X.-M. Mei, Note on the residues of the singularities of a Riemannian foliation, Proc. Amer. Math. Soc., 89:359-366, 1983. MR712652 (85g:53042)

[53] K. Millett, Compact foliations, in Differential topology and geometry (Proc. Colloq., Dijon, 1974), Lect. Notes in Math. Vol. 484, Springer-Verlag, New York and Berlin, 1975, pages 277-287. MR0391122(52:11944)

[54] P. Molino, Étude des feuilletages transversalement complets et applications, Ann. Sci. École Norm. Sup. (4), 10:289-307, 1977. MR0458446 (56:16649)

[55] P. Molino, Géométrie globale des feuilletages riemanniens, Nederl. Akad. Wetensch. Indag. Math., 44:45-76, 1982. MR653455 (84j:53043)

[56] P. Molino, Riemannian foliations, Translated from the French by Grant Cairns, with appendices by Cairns, Y. Carrière, É. Ghys, E. Salem and V. Sergiescu, Birkhäuser Boston Inc., Boston, MA, 1988. MR932463 (89b:53054)

[57] P. Molino, Orbit-like foliations, in Geometric Study of Foliations, Tokyo 1993 (eds. Mizutani et al.), World Scientific Publishing Co. Inc., River Edge, N.J., 1994, 97-119. MR.1363720 (97e:57030)

[58] I. Moerdijk and J. Mrčun, Introduction to foliations and Lie groupoids, Cambridge Studies in Advanced Mathematics, Vol. 91, Cambridge University Press, Cambridge, 2003. MR2012261 (2005c:58039)

[59] R.S. Palais, Lusternik-Schnirelman theory on Banach manifolds, Topology, 5:115-132, 1966. MR0259955 (41:4584)

[60] R. Palais and C.-L. Terng, Critical point theory and submanifold geometry, Lect. Notes in Math. Vol. 1353, Springer-Verlag, Berlin, 1988. MR972503 (90c:53143)

[61] K. Richardson, The asymptotics of heat kernels on Riemannian foliations, Geom. Funct. Anal., 8:356-401, 1998. MR.1616151 (99e:58188)

[62] K. Richardson, The transverse geometry of G-manifolds and Riemannian foliations, Illinois J. Math., 45:517-535, 2001. MR 1878616 (2002k:53041)

[63] É. Salem, Une généralisation du théorème de Myers-Steenrod aux pseudogroupes d'isométries, Ann. Inst. Fourier (Grenoble), 38:185-200, 1988. MR949015 (89i:58166)

[64] I. Satake, On a generalization of the notion of manifold, Proc. Nat. Acad. Sci. U.S.A., 42:359-363, 1956. MR0079769 (18:144a)

[65] J.-M. Souriau, Groupes différentiels, In Differential geometrical methods in mathematical physics (Proc. Conf., Aix-en-Provence/Salamanca, 1979), Lect. Notes in Math. Vol. 836, Springer-Verlag, Berlin, 1980, pages 91-128. MR607688 (84b:22038)

[66] P. Stefan, Accessible sets, orbits, and foliations with singularities, Proc. London Math. Soc. (3), 29:699-713, 1974. MR0362395 (50:14837)

[67] P. Stefan, Integrability of systems of vector fields, J. London Math. Soc. (2), 21:544-556, 1980. MR577729 (81h:49026)

[68] T. tom Dieck, Transformation groups, de Gruyter Studies in Mathematics, Vol. 8, 1987. MR889050 (89c:57048)

[69] R.A. Wolak, Basic forms for transversely integrable singular Riemannian foliations, Proc. Amer. Math. Soc., 128:1543-1545, 2000. MR.1662230 (2000j:53034)

[70] R.A. Wolak, Critical leaves of basic functions for a singular Riemannian foliation, preprint 2002.

[71] K. Yamato, Sur la classe caractéristique exotique de Lazarov-Pasternack en codimension 2, C. R. Acad. Sci. Paris Sér. A-B, 289:A537-A540, 1979. MR557268 (81f:57029) 
[72] K. Yamato, Sur la classe caractéristique exotique de Lazarov-Pasternack en codimension 2. II, Japan. J. Math. (N.S.), 7:227-256, 1981. MR729436 (85e:57032)

Department of Mathematics, University of Illinois at Chicago, 322 SeO (m/c 249), 851 S. Morgan Street, Chicago, Illinois 60607-7045

E-mail address: hurder@uic.edu

Mathematisches Institut, Universität zu Köln, Weyertal 86-90, 50931 Köln, Germany

E-mail address: dtoeben@math.uni-koeln.de 